\title{
Alterations in the transcription factors GntR1 and RamA enhance the growth and central metabolism of Corynebacterium glutamicum
}

Wang, Zhihao; Liu, Jianming; Chen, Lin; Zeng, An-Ping; Solem, Christian; Jensen, Peter Ruhdal

Published in:

Metabolic Engineering

Link to article, DOI:

10.1016/j.ymben.2018.05.004

Publication date:

2018

Document Version

Peer reviewed version

Link back to DTU Orbit

Citation (APA):

Wang, Z., Liu, J., Chen, L., Zeng, A-P., Solem, C., \& Jensen, P. R. (2018). Alterations in the transcription factors GntR1 and RamA enhance the growth and central metabolism of Corynebacterium glutamicum. Metabolic Engineering, 48, 1-12. https://doi.org/10.1016/j.ymben.2018.05.004

\section{General rights}

Copyright and moral rights for the publications made accessible in the public portal are retained by the authors and/or other copyright owners and it is a condition of accessing publications that users recognise and abide by the legal requirements associated with these rights.

- Users may download and print one copy of any publication from the public portal for the purpose of private study or research.

- You may not further distribute the material or use it for any profit-making activity or commercial gain

- You may freely distribute the URL identifying the publication in the public portal 


\section{Author's Accepted Manuscript}

Alterations in the transcription factors GntR1 and RamA enhance the growth and central metabolism of Corynebacterium glutamicum

Zhihao Wang, Jianming Liu, Lin Chen, An-Ping Zeng, Christian Solem, Peter Ruhdal Jensen

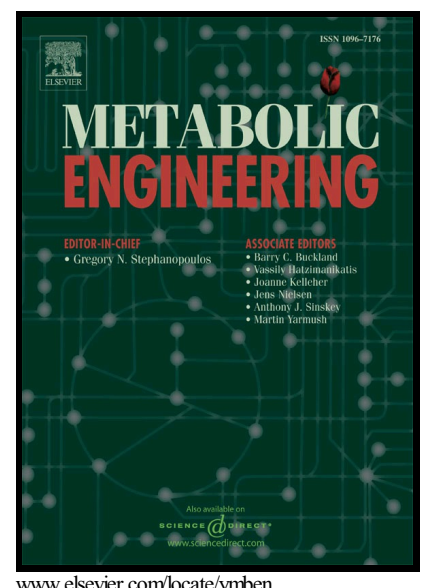

PII:

S1096-7176(18)30117-4

DOI: $\quad$ https://doi.org/10.1016/j.ymben.2018.05.004

Reference: YMBEN1402

To appear in: Metabolic Engineering

Received date: 12 March 2018

Accepted date: 7 May 2018

Cite this article as: Zhihao Wang, Jianming Liu, Lin Chen, An-Ping Zeng, Christian Solem and Peter Ruhdal Jensen, Alterations in the transcription factors GntR1 and RamA enhance the growth and central metabolism of Corynebacterium glutamicum, Metabolic Engineering, https://doi.org/10.1016/j.ymben.2018.05.004

This is a PDF file of an unedited manuscript that has been accepted for publication. As a service to our customers we are providing this early version of the manuscript. The manuscript will undergo copyediting, typesetting, and review of the resulting galley proof before it is published in its final citable form. Please note that during the production process errors may be discovered which could affect the content, and all legal disclaimers that apply to the journal pertain. 


\title{
ACCEPTED MANUSCRIPT
}

Alterations in the transcription factors GntR1 and RamA enhance the growth and central metabolism of Corynebacterium glutamicum

Zhihao Wang ${ }^{\mathrm{a}}$, Jianming Liu ${ }^{\mathrm{a}}$, Lin Chen ${ }^{\mathrm{b}}$, An-Ping Zeng ${ }^{\mathrm{b}}$, Christian Solem ${ }^{\mathrm{a}^{*}}$, Peter Ruhdal Jensen ${ }^{\mathrm{a}^{*}}$

${ }^{a}$ The National Food Institute, Technical University of Denmark, Kongens Lyngby 2800, Denmark;

${ }^{\mathrm{b}}$ Institute of Bioprocess and Biosystems Engineering, Hamburg University of Technology, Hamburg,

Germany.

Christian Solem (chso@food.dtu.dk)

Peter Ruhdal Jensen (perj@food.dtu.dk)

${ }^{*}$ Corresponding authors. National Food Institute, Technical University of Denmark, Building 221, Anker Engelundsvej 1, 2800, Kongens Lyngby, Denmark.

\begin{abstract}
Evolution, i.e. the change in heritable characteristics of biological populations over successive generations, has created the diversity of life that exists today. In this study we have harnessed evolution to create faster growing mutants of Corynebacterium glutamicum, i.e. to debottleneck growth rate of this highly important industrial workhorse. After approximately 1500 generations of Adaptive Laboratory Evolution (ALE) in defined minimal medium with glucose, we obtained faster growing mutants with specific growth rate as high as $0.64 \mathrm{~h}^{-1}$ as compared with $0.45 \mathrm{~h}^{-1}$ for the wild type, and this $42 \%$ improvement is the highest reported for C. glutamicum to date. By genome resequencing and inverse metabolic engineering, we were able to pinpoint two mutations contributing to most of the growth improvement, and these resided in the transcriptional regulators GntR1 (gntR1-E70K) and RamA (ramA-A52V). We confirmed that the two
\end{abstract}




\section{ACCEPTED MANUSCRIPT}

mutations lead to alteration rather than elimination of function, and their introduction in the wild-type background resulted in a specific growth rate of $0.62 \mathrm{~h}^{-1}$. The glycolytic and pentose phosphate pathway fluxes had both increased significantly, and a transcriptomic analyses supported this to be associated with increased capacity. Interestingly, the observed fast growth phenotype was not restricted to glucose but was also observed on fructose, sucrose and xylose, however, the effect of the mutations could only be seen in minimal medium, and not rich BHI medium, where growth was already fast. We also found that the mutations could improve the performance of resting cells, under oxygen-deprived conditions, where an increase in sugar consumption rate of around $30 \%$ could be achieved. In conclusion, we have demonstrated that it is feasible to reprogram $C$. glutamicum into growing faster and thus enhance its industrial potential. Keywords: Corynebacterium glutamicum; adaptive evolution; fast growth; genomic analysis; GntR1; RamA

\section{Introduction}

The specific growth rate is a key parameter, which needs to be considered when assessing the suitability of a particular microorganism for industrial use. For growth-coupled production processes, fast growth is an obvious advantage (Feist et al., 2010; Grünberger et al., 2013; Liu et al., 2016a, 2017), but also in general fast growth is desirable as it allows for a high cell density and high productivity to be attained more rapidly. Finally a fast growth phenotype is desirable to compensate for the reduction in growth rate often seen when harnessing the metabolic machinery for production (Lee and Kim, 2015; Liu et al., 2016b, c). Fast growing organism do exist, however, they are not always suited for industrial production, e.g. due to safety reasons, higher nutritional requirements, etc, and sometimes a better option may be to improve growth properties a candidate more suited to industrial production. Adaptive laboratory evolution (ALE), in combination with various other tools, has become a powerful approach for studying how microorganisms adapt genetically to improve fitness, over time, to certain conditions or changes in environmental parameters (Mahr et al., 2015; Oide et al., 2015; Takeno et al., 2013). A classic example is the work of Richard Lenski and co-workers, who studied adaptation of Escherichia coli to growth in a glucose-limited minimal medium over 40,000 generations (Barrick et al., 2009). ALE has also been demonstrated to be extremely useful for optimizing 


\section{ACCEPTED MANUSCRIPT}

properties of microorganisms, e.g. metabolic imbalances, inhibitor tolerance, thermal tolerance and ability to use certain carbon (Caspeta et al., 2014; Cheng et al., 2014; Hong et al., 2011; Ibarra et al., 2002; Jakob et al., 2007; Long et al., 2017)

One important reason that has stimulated the use of ALE in recent years is the development of reliable sequencing technologies, which has simplified the task of identifying the correlations between phenotype and genotype (Albert et al., 2005; Dettman et al., 2012; Herring et al., 2006). Cheap and fast access to genome sequences of adapted mutants has made it possible to apply inverse metabolic engineering for creating superior cell factories devoid of the many mutations that inevitably are introduced in the course of ALE (Bailey et al., 1996; Jin et al., 2005). In ALE, beneficial mutations normally emerge after hundreds to thousands of generations, depending on microbe and cultivation conditions (James Winkler, Luis H. Reyes, 2013). However, under some conditions, e.g. during starvation, certain bacteria have been reported to mutate more rapidly, which increases the efficiency of ALE for obtaining useful mutants (Kim et al., 2001). The actinobacterium Corynebacterium glutamicum, is an important and established industrial workhorse due to its robustness and metabolic flexibility, and this organism has been engineered into producing a broad range of useful compounds, such as amino acids, organic acids, alcohols and even non-natural compounds (Becker and Wittmann, 2015, 2012). C. glutamicum has quite simple growth requirements and grows well in chemically defined minimal medium containing a suitable carbon source. Various kinds of carbohydrates, e.g. glucose, fructose, and sucrose, or organic acids like acetate, pyruvate and lactate can readily be used by C. glutamicum (Ikeda, 2012). What appears to be highly unusual for this bacterium is that it does not appear to have traditional catabolite control repression (CCR) mechanisms, and thus different common carbon substrates can be metabolized simultaneously (Moon et al., 2007).

Various defined media containing glucose have been described for C. glutamicum, optimized in terms of growth rate over the past decades, e.g. MM (Liebl et al., 1989), CGXII (Keilhauer et al., 1993) and similar media (Bäumchen et al., 2007; Kind et al., 2010). Recently, Noack and colleagues achieved the fastest growth $\left(0.61 \mathrm{~h}^{-1}\right)$ ever reported for $C$. glutamicum in glucose minimal medium at the single cell level in a microfluidic perfusion system (Grünberger et al., 2013). Subsequent work revealed that the cause for the fast growth was due to co-metabolism of protocatechuic acid (PCA) and glucose (Unthan et al., 2014). These 


\section{ACCEPTED MANUSCRIPT}

results indicate that there are bottlenecks controlling how fast $C$. glutamicum can grow on glucose, and this is also supported by the finding that sucrose supports an even faster growth than glucose (Wang et al., 2016). In C. glutamicum several regulators are involved in governing central metabolism. The DeoR-type regulator SugR is considered to be a repressor of several PTS genes, however it has been found that its inactivation only has a marginal effect on the phenotype (Engels and Wendisch, 2007). Yukawa et al., demonstrated, by over-expressing several glycolytic enzymes, that the glycolytic flux could be enhanced, however, only for oxygen deprived resting cells (non-growing), and not under normal aerobic growth conditions (Yamamoto et al., 2012). In our previous studies we found that by perturbing the fructose operon, we could increase the growth rate of $C$. glutamicum, on glucose, by $10 \%$ and we found that this increase in growth rate correlated with an accumulation of intracellular fructose-1-phosphate (Wang et al., 2016). Besides these studies, there have been relatively few efforts focusing on finding factors controlling glucose metabolism in C. glutamicum, which makes it an interesting topic to study, in particular in the light of the potential implications it could have for bio-based production using this organism.

The objective of the current study is thus to shed additional light on factors controlling the growth and metabolism of carbohydrates. First we adapt the wild-type C. glutamicum strain ATCC 13032 on glucose minimum medium for more than 1500 generations and isolate several faster growing mutants. We carefully characterize one of these mutants through growth experiments, genome sequencing, and transcriptome analysis. Single mutations, contributing to most of the faster growth phenotype are identified in the two transcriptional regulators GntR1 and RamA, and we demonstrate that these mutations result in faster metabolism of glucose and other sugars, both by growing and resting cells.

\section{Material and methods}

\section{Bacterial strains and medium.}

All bacterial strains and plasmids used in this study are listed in Supplementary Table 1. For molecular cloning, E. coli strains were aerobically grown at $37^{\circ} \mathrm{C}$ in Luria-Bertani (LB) broth, and C. glutamicum 


\section{ACCEPTED MANUSCRIPT}

strains were aerobically grown at $30^{\circ} \mathrm{C}$ in BHI broth (Sigma, Prod. No.53286). When appropriate, the solid medium was supplemented with kanamycin $(50 \mu \mathrm{g} / \mathrm{ml}$ for E. coli, $25 \mu \mathrm{g} / \mathrm{ml}$ for C. glutamicum), or $10 \%$ sucrose. For physiological characterization of $C$. glutamicum strains, a defined minimal medium (MM) was used which was modified by adding MOPS buffer, supplemented with $11 \mathrm{mg} / \mathrm{L}$ catechol, $4.6 \mathrm{mg} / \mathrm{L}$ thiamin, $11.3 \mathrm{mg} / \mathrm{L}$ calcium pantothenate, $5.7 \mathrm{mg} / \mathrm{L}$ nicotinic acid, $1.3 \mathrm{mg} / \mathrm{L}$ biotin (Wang et al., 2014). For comparisons, the CGXII medium containing $30 \mathrm{mg} / \mathrm{L}$ protocatechuic acid (iron chelator) was prepared as well (Unthan et al., 2014).

\section{Cultivation conditions and adaptive evolution}

In general, for growth experiments, cells from frozen glycerol stock cultures were plated on BHI agar and incubated overnight at $30^{\circ} \mathrm{C}$. Single colonies were inoculated into $20 \mathrm{ml}$ minimal medium in a $250-\mathrm{ml}$ shake flask and grown for $15-20 \mathrm{~h}$ to obtain the pre-culture. The main cultivation was performed in $50 \mathrm{ml}$ minimal medium in a 500-ml shake flask for $24 \mathrm{~h}$. All shake flask cultivations were carried out at $30^{\circ} \mathrm{C}$ and $220 \mathrm{rpm}$ in a rotary shaker and in biological triplicates. Cell growth was monitored by measuring the optical density at $600 \mathrm{~nm}\left(\mathrm{OD}_{600}\right)$ of the culture broth with a UV1800 spectrophotometer (Shimadzu). The dry cell weight was

calculated according to the following formula: $\mathrm{DCW}(\mathrm{g} / \mathrm{L})=\mathrm{OD}_{600} \times 0.3$ (Shinfuku et al., 2009). The measured correlations between DCW and $\mathrm{OD}_{600}$ for different strains are shown in Fig. S7.

The adaptive laboratory evolution (ALE) was carried out in $150 \mathrm{~mL}$ shake flasks containing $15 \mathrm{~mL}$ minimal medium with $1 \%$ glucose at $220 \mathrm{rpm}$ and $30^{\circ} \mathrm{C}$. The biomass $\left(\mathrm{OD}_{600}\right)$ was measured every $24 \mathrm{hrs}$ and $150 \mu \mathrm{l}$ of the culture was transferred into a fresh medium. This process was continued for about 7 months (approximately 1500 generations). Once a week, a culture sample was withdrawn and stored at $-80^{\circ} \mathrm{C}$ for future analysis. Isolates were subsequently obtained from these frozen stocks at week 10 (approximately 500 generations), week 20 (approximately $\sim 1000$ generations), and week 30 (approximately $\sim 1500$ generations), respectively, by plating on solid medium.

For the resting cells experiments (oxygen deprivation), $100 \mathrm{ml}$ of over-night cultures (BHI supplemented with $1 \%$ glucose) were harvested by centrifugation at room temperature (6000 rpm, $5 \mathrm{~min}$ ). The cell pellets 


\section{ACCEPTED MANUSCRIPT}

were washed twice with $0.9 \% \mathrm{NaCl}$ after which an appropriate amount of washed cells were re-suspended in 100-ml of MM with 2\% glucose in bottles filled to the rim. The cell density obtained corresponded to an $\mathrm{OD}_{600}$ of approximately 20 . Cell suspensions were incubated at $30^{\circ} \mathrm{C}$ under static conditions with slow magnetic stirring (no oxygen could enter). To prevent a drop in $\mathrm{pH}$, the medium was supplemented $200 \mathrm{mM}$ of MOPS buffer.

\section{Recombinant DNA techniques and strain construction}

All molecular manipulations including PCR amplification, purification, enzymatic digestion, ligation, preparation of competent cells and electroporation were performed according to standard protocols (Sambrook J, Fritsch EF, 1989). All primers used in the current study are listed in Supplementary Table 2. For the re-construction of $r a m A^{\text {mut }}$ in the WT or $\Delta$ CGP3 strain kindly donated by Prof. Frunzke (Baumgart et al., 2013), the plasmid pK18-ramA ${ }^{\text {mut }}$ was transferred by electroporation into the competent cells and the transformation mixture was plated on BHI agar containing $25 \mu \mathrm{g} / \mathrm{ml}$ kanamycin. After a two-step double homologous recombination event (Becker et al., 2011), colonies being both kanamycin-sensitive and sucrose-resistant were isolated and verified by Sanger sequencing (Macrogen, Korea) to possess the particular mutation. The plasmid pK18-ram $A^{\text {mut }}$ contained two fragments, each approximately 750 -bp in size, corresponding to the upstream and downstream regions of the single nucleotide variation $(\operatorname{cg} 2831: 155 \mathrm{C}>\mathrm{T})$ respectively. The two PCR fragments were amplified and inserted into the empty vector pK18mobsacB, which had been digested using SmaI according to the Gibson Assembly procedure (Gibson et al., 2009). The assembled mixture was then transformed into E. coli TOP10, and the resulting plasmid was designated as pK18-ramA ${ }^{\mathrm{mut}}$. Similarly, the plasmids pK18-gntRI ${ }^{\mathrm{mut}}(\operatorname{cg} 2783: 208 \mathrm{G}>\mathrm{A}), \mathrm{pK} 18-\Delta g n t R 1$, and pK18- $\Delta r a m A$ were constructed in the same way, while other re-constructed mutants such as $g n t R I^{\mathrm{mut}}, \Delta g n t R l, \Delta r a m A$, and DKI ( $\left.g n t R I^{\mathrm{mut}} r a m A^{\mathrm{mut}}\right)$ were created using the plasmids mentioned above respectively.

\section{Sample preparation and substrate quantification}




\section{ACCEPTED MANUSCRIPT}

For isolation of chromosomal DNA from C. glutamicum, $2 \mathrm{ml}$ of an exponentially growing culture $\left(\mathrm{OD}_{600}\right.$ around 1, BHI medium) were harvested by centrifugation. The cell pellet was re-suspended in $500 \mu 10.9 \%$ $\mathrm{NaCl}$ and treated for two hours, at $37^{\circ} \mathrm{C}$, with $30 \mu \mathrm{l}$ Lysozyme $(5 \mathrm{mg} / \mathrm{ml})$ (Sigma Aldrich), after which $15 \mu \mathrm{l}$ proteinase $\mathrm{K}(1 \mathrm{mg} / \mathrm{ml})$ was added and the incubation was extended for an additional two hours. Afterwards, the chromosomal DNA was extracted as described by Katsumata (Katsumata et al., 1984).

For isolation of RNA from $C$. glutamicum, exponentially growing cells $\left(\mathrm{OD}_{600}\right.$ around 1$)$ in minimal medium with $1 \%$ glucose were harvested and centrifuged $\left(7000^{*} \mathrm{~g}, 5 \mathrm{~min}\right.$ at $\left.4^{\circ} \mathrm{C}\right)$. The cell pellet from $25 \mathrm{ml}$ culture was washed twice in $0.9 \% \mathrm{NaCl}$ and then resuspended in $200 \mu \mathrm{l}$ Solution I $(0.3 \mathrm{M}$ sucrose and $10 \mathrm{mM} \mathrm{NaAc}$, pH 4.8) and $200 \mu \mathrm{l}$ preheated Solution II (2\% SDS and 0.01 M NaAc, pH 4.8). Next, RNA was extracted from the homogenized cell lysate using the phenol/acetate/chloroform method described previously (Chan et al., 2014). Finally, the precipitated RNA was dissolved in DEPC-treated water and stored at $-80^{\circ} \mathrm{C}$. For intracellular ATP and ADP measurements, $600 \mu \mathrm{l}$ of exponentially growing culture (MM with $1 \%$ glucose) was withdrawn at an $\mathrm{OD}_{600}$ around 1 and immediately vortexed with same volume of $80^{\circ} \mathrm{C}$ phenol and glass beads (106 $\mu \mathrm{m}$ in diameter, Sigma). Next, the cell extract was prepared as previously described (Koebmann et al., 2002b). The concentrations of ATP and ADP were then measured using the luciferinluciferase ATP Determination Kit (A22066, Thermo Fisher Scientific), as described previously (Liu et al., 2016b).

For determining fermentation products and various sugars an Ultimate 3000 HPLC system (Dionex, Sunnyvale, USA) equipped with an Aminex HPX-87H column (Bio-Rad, USA) and a Shodex RI-101 detector (Showa Denko KK, Tokyo, Japan) was used. The column oven temperature was set to $60^{\circ} \mathrm{C}$, and the mobile phase was $5 \mathrm{mM}$ sulfuric acid with a flow rate of $0.5 \mathrm{ml} / \mathrm{min}$.

\section{Whole genome re-sequencing}

Genome re-sequencing was carried out by BGI-Tech (HongKong), using Illumina Hiseq-4000 equipment, and on average, more than 120 -fold coverage of the genome was achieved. The resulting data were 100-bp paired end reads, which were trimmed and assembled using Bowtie2 developed by the Langmead Lab (Langmead and Salzberg, 2012). The C. glutamicum ATCC 13032 (GenBank accession number: 
NC_006958) genome was used as reference. Variants (SNPs, short indels, and large deletions) were called based on the Bowtie2 assembled data using GATK toolkit by Broad Institute (DePristo et al., 2011). The identified variants were verified by using conventional sequencing.

\section{Transcriptomics analysis}

The transcriptomics analysis was accomplished using Illumina HiSeq-4000 based on the service of RNA-Seq Quantification library at BGI-Tech (HongKong). Datasets consisted of 10M reads per sample with a 50-bp read length. Afterwards, the RNA-seq data was assembled and analyzed by comparing with the translational region of annotated DNA sequence data of reference (GCA_000196335.1.29) using HiSat2 (Kim et al., 2015) and HTSeq (Anders et al., 2015). The estimation of fold change and other statistical analysis was performed using the DESeq2 package in R (Love et al., 2014). The GO enrichment analysis was carried out by using the function enrichKEGG in the clusterProfiler (R-package) (Yu et al., 2012).

\section{${ }^{13} \mathrm{C}$-metabolic flux analysis}

To quantify the intracellular metabolic fluxes, ${ }^{13} \mathrm{C}$-MFA was performed. The WT and DKI strains were grown in MM medium with $0.5 \%$ glucose $\left(80 \% 1-{ }^{13} \mathrm{C}\right.$ and $\left.20 \% \mathrm{U}^{-13} \mathrm{C}\right)$ in duplicates. The samples were prepared based on the protocol by Zamboni et al. (2009) and the parameters for GC-MS measurements can be seen in Supplementary Table 3. Here we assumed the cellular composition was stable so that any potential adaptations in anabolic fluxes that might have occurred were neglected in the study.

The metabolic flux model used for ${ }^{13} \mathrm{C}-\mathrm{MFA}$ is based on the $C$. glutamicum model described previously and the simulations were performed using OpenFLUX2 software (Shupletsov et al., 2014). The absolute fluxes in $\mathrm{mmol} / \mathrm{gDW} / \mathrm{h}$ are calculated by multiplication with the specific glucose uptake rate (Kromer et al. 2004). At convergence, accurate $95 \%$ confidence intervals were computed for most estimated fluxes by evaluating the sensitivity of the minimized SSR to flux variations. Precision or standard deviations (SD) of the estimated fluxes were determined by the following equation: $\mathrm{SD}=\left(\right.$ Flux $\left.x_{\text {upper bound } 95 \%}-F l u x_{\text {lower bound } 95 \%}\right) / 4$. 


\section{ACCEPTED MANUSCRIPT}

\section{Results}

\section{Experimental adaption of $C$. glutamicum in minimal glucose medium}

It remains interesting to elucidate the limiting factors that control the specific growth rate of a microorganism, in particular the ones that have a high industrial relevance. In this study, we have tried to address this question for $C$. glutamicum by using ALE. The wild-type strain C. glutamicum ATCC13032 was grown for an extended period of time, 7 months, in a defined minimal medium containing glucose. From culture samples obtained after 500, 1,000 and 1,500 generations of growth we isolated colonies which were designated as G5H, G10H, and G15H (Fig.1). Characterization revealed that all isolates grew faster than the wild-type strain (WT) by 31,37 , and $42 \%$ respectively, and the faster growth was found to correlate to a similar increase in specific glucose consumption rate $(6.73 \pm 0.35 \sim 6.87 \pm 0.24 \mathrm{mmol} / \mathrm{g} \cdot \mathrm{DCW} / \mathrm{h}$ or $33 \% \sim$ $37 \%$ increase). The biomass yield on glucose and final cell density (OD600) remained unaffected for all strains.
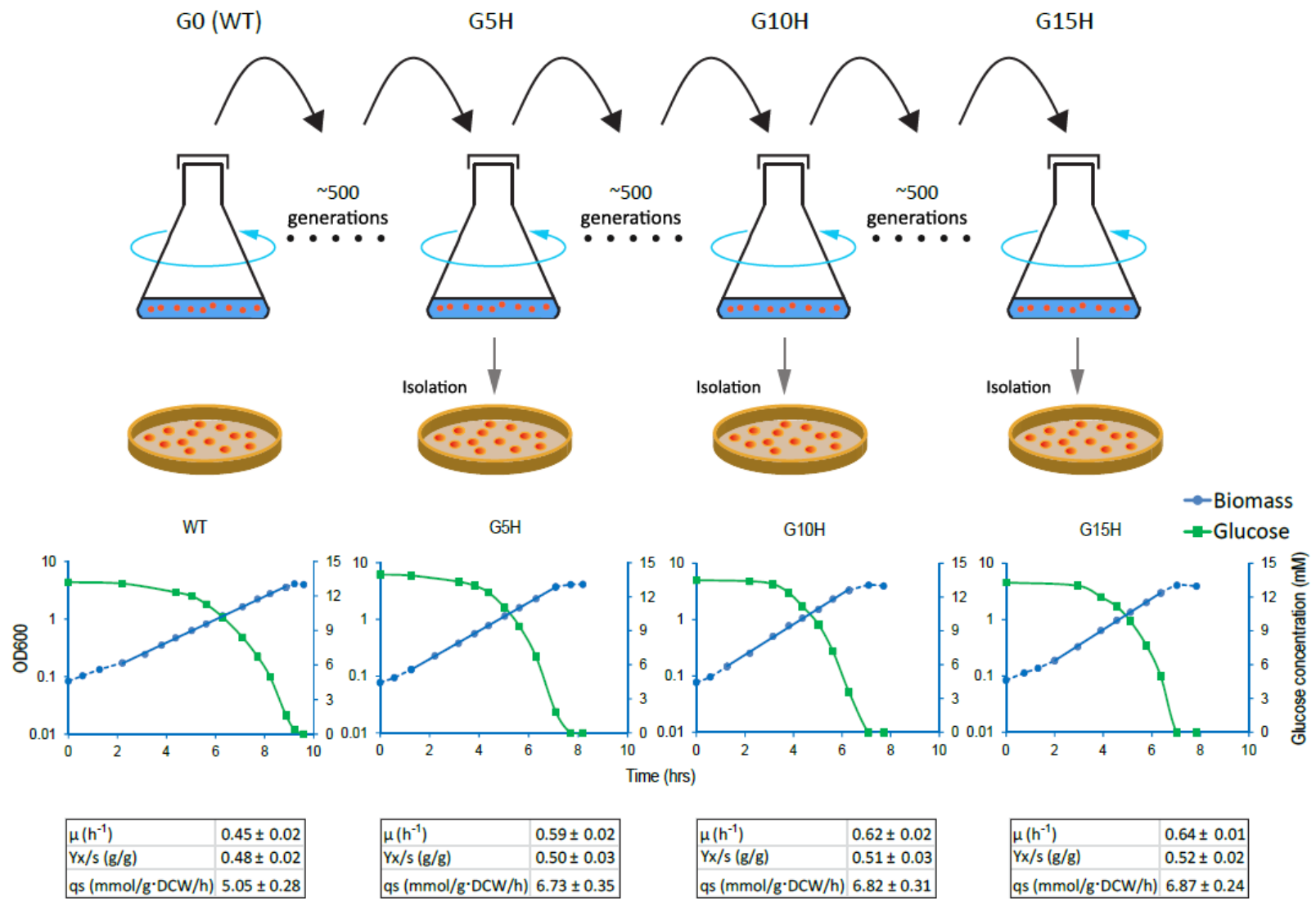


\section{ACCEPTED MANUSCRIPT}

Fig.1 Schematic showing the procedure used for ALE and its outcome. Serial propagation in shaking flask leads to selection of mutants with increased growth rates. Strains were isolated every 500 generations and designated as G5H (500 gen), G10H (1,000 gen), and G15H (1,500 gen). Physiological characterization was performed in biological triplicates and the presented data $\mu$ (specific growth rate), $Y_{x / s}$ (biomass yield), and $q_{s}$ (glucose consumption rate), were averages based on these independent experiments.

\section{Whole genome re-sequencing of the isolated adaptive mutants}

To identify the mutations responsible for the faster growth phenotype, we sequenced the genomes of the WT, G5H, G10H, and G15H. As shown in Supplementary Fig. S1, all three adapted mutants were found to lack a $180 \mathrm{kbp}$ genomic fragment (cg1890 - cg2065), which previously has been identified as the prophage CGP3 cluster (cg1890 - cg2071) (Baumgart et al., 2013; Kalinowski et al., 2003).

Although the end part of the CGP3 cluster was conserved in our mutants, dozens of mutations were observed in the genes $c g 2066$ and $c g 2069$. Aside from mutations in the prophage region or in the intergenic region (cg1501/1502, cg1562/1563, cg1889/1890), only four additional mutations were found, and these were shared between the three isolates: $\mathrm{C}$ to A exchange at position 83 in $\operatorname{cg} 0712$ (hypothetical protein), leading to amino acid replacement of Thr-28 by Asn; G to A exchange at position 208 in cg2783 (GntR1: GntR family transcriptional regulator), leading to amino acid replacement of Glu-70 by Lys; $\mathrm{C}$ to $\mathrm{T}$ exchange at position 155 in $\operatorname{cg} 2831$ (RamA: LuxR family transcriptional regulator), leading to amino acid replacement of Ala-52 by Val; A to $\mathrm{G}$ exchange at position 878 in $\operatorname{cg} 3165$ (hypothetical protein), leading to amino acid replacement of Asp-293 by Gly (Fig. 2). A summary of the genomic re-sequencing results can also be seen in Supplementary Data 1. 


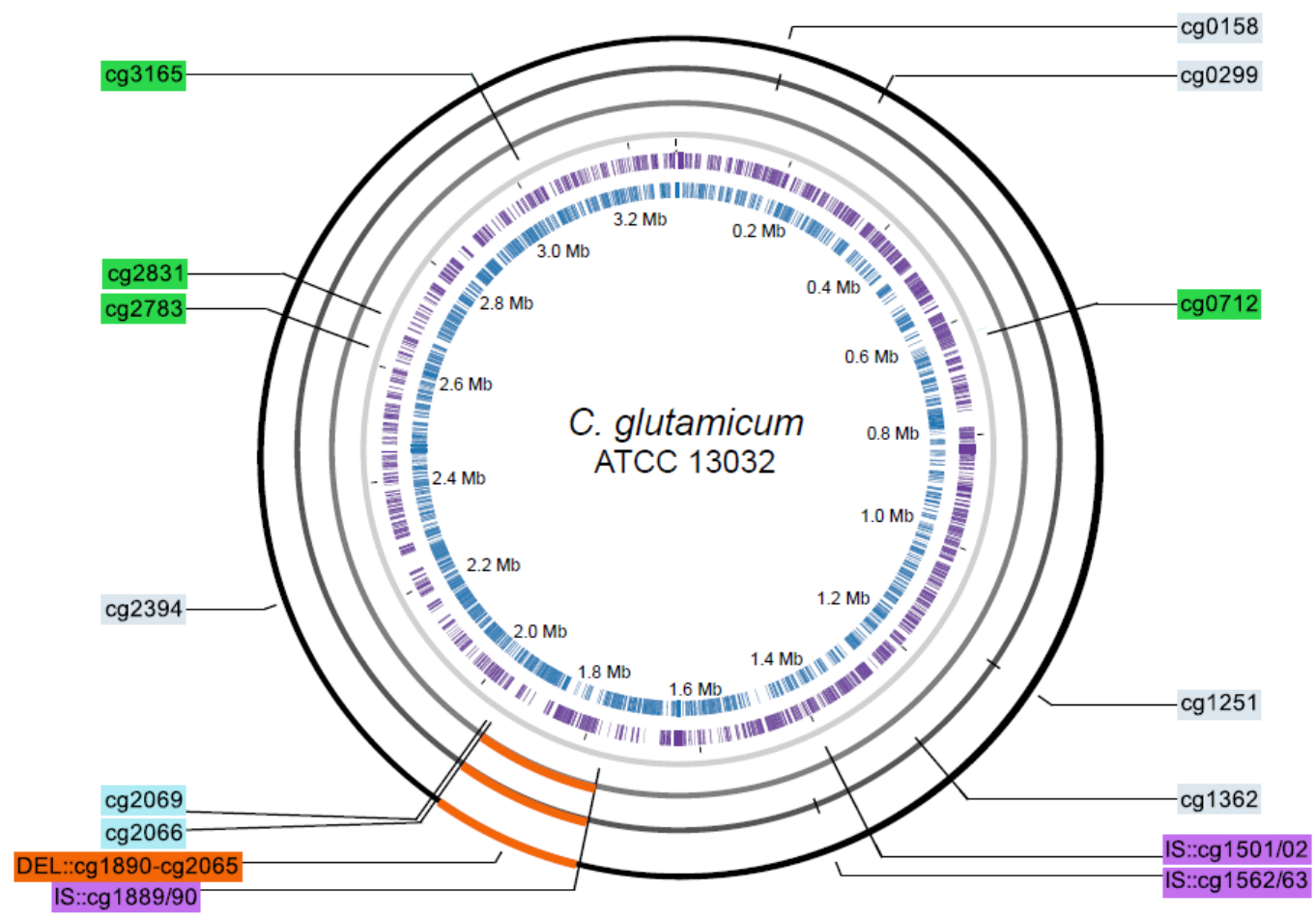

Isolate sequenced from generation..

Evolved mutations...

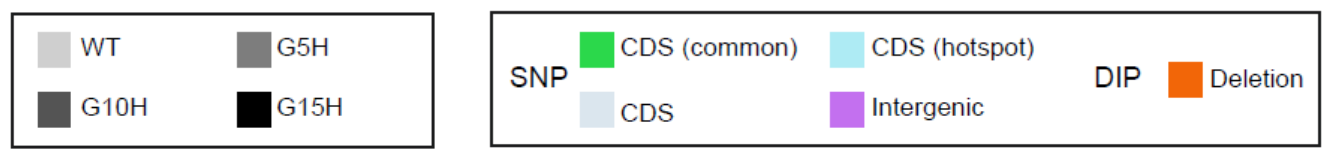

Fig. 2 Mutations found by sequencing the three faster growing mutants obtained in the ALE experiment. The four rings, from outer to inner, represent the genomes of $\mathrm{G} 15 \mathrm{H}, \mathrm{G} 10 \mathrm{H}$ and $\mathrm{G} 5 \mathrm{H}$ sampled at 1,500, 1,000, and 500 generations respectively, and that of the WT. The innermost circle (blue) indicates the genome position in megabase pairs (Mb). Also indicated are the SNP's, which are highlighted in either green, light blue, light grey when they occur in genes or purple when located in intergenic regions. Noticeably, the light blue highlighting indicates many mutations in genes cg2066 and cg2069 (hotspots). The region indicated in orange corresponds to the prophage region (CGP3), which is missing in all three ALE strains. SNP: single nucleotide polymorphism; DIP: deletion/insertion polymorphism.

\section{Identifying the causative mutations.}

To confirm the involvement of the four common mutations discovered above, we first introduced each of them in the WT background. Only the mutation in $\operatorname{ramA}$ seemed to have an effect, however, the effect was a noticeable 20\% increase in specific growth rate (Supplementary Fig. S2). Previously it has been reported that when the prophage region is deleted ( $\triangle \mathrm{CGP} 3)$, the cell phenotype is insignificantly affected (Baumgart et al., 
2013; Unthan et al., 2015). Since all the faster growing mutants had a large truncation and several mutations in this region, we decided to assess the effect of the four mutations in a $\triangle \mathrm{CGP} 3$ strain background.

Consistent with previous findings, the $\triangle \mathrm{CGP} 3$ strain grew as fast as the WT on glucose. Surprisingly, in the $\triangle$ CGP3 background, both the $\operatorname{ramA}$ (LUXR: $\triangle \mathrm{CGP} 3$ with ramA-A52V) and the gntR1 mutation (GNTR: $\Delta$ CGP3 with gntRl-E70K) had positive effects on growth. The growth rate was increased by $22 \%$ due to the $\operatorname{ramA}$ mutation $\left(0.55 \pm 0.02 \mathrm{~h}^{-1}\right)$ and by $8.8 \%\left(0.48 \pm 0.01 \mathrm{~h}^{-1}\right)($ Fig. $3 \mathrm{~A})$ when the gntRl mutation was introduced. We further found that the effects of the single mutations were additive, and the double knock-in strain, DKI, grew almost as fast $\left(0.62 \pm 0.02 \mathrm{~h}^{-1}\right)$ as the adapted mutants. The increase in specific growth rate also correlated well with an equally large increase in glucose consumption rate, $36 \%$ higher than that of the WT $(6.92 \pm 0.32 \mathrm{mmol} / \mathrm{g} \cdot \mathrm{DCW} / \mathrm{h})$. More details can be seen in Supplementary Table 4 .

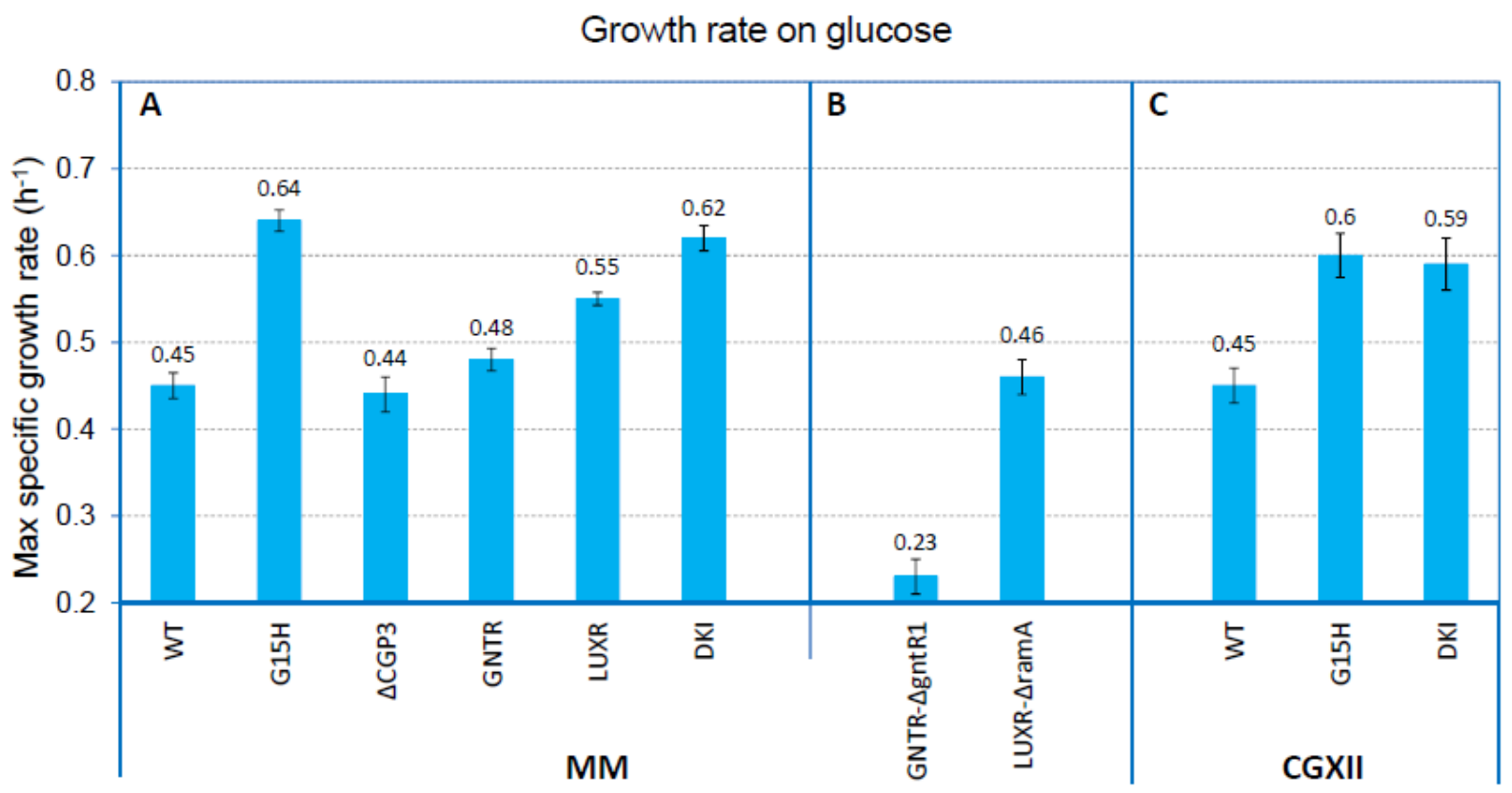

Fig. 3 A comparison of the specific growth rates of adapted or reconstructed mutants on glucose. A: Specific growth rates on MM for the following strains are shown: G15H (mutant isolated in ALE experiment after 1,500 generations); $\triangle \mathrm{CGP} 3$ (the WT lacking the CGP3 region); GNTR ( $\triangle \mathrm{CGP3}$ with gntR1 mutant); LUXR ( $\triangle \mathrm{CGP3}$ with ramA mutant); DKI ( $\triangle \mathrm{CGP} 3$ with the gntR1 and ramA mutations). B: Specific growth rates on MM for GNTR- $\Delta g n t R 1$ and LUXR-AramA were determined as well. C: As a complementary test, the growth rates of WT, G15H, and DKI strain are also compared on the more common defined medium CGXII (Unthan et al., 2014). The growth rates and standard deviations were calculated based on data from three independent experiments. 
It has previously been found that a $\operatorname{ramA}(\mathrm{cg} 2831)$ knock-out mutant is unable to grow on acetate as sole carbon source, but that it grows just as well as the WT on glucose (Cramer A, et al., 2006). We found that the LUXR strain, which carries a mutated ramA-A52V gene, is able to grow on acetate (Supplementary Fig. S3), and this indicates that the mutation does not lead to inactivation but rather changed function. To prove this, the mutated ramA-A52V gene was deleted from LUXR. The resulting strain, LUXR- $\Delta r a m A$, indeed was neither able to grow on acetate, nor display improved growth on glucose (Supplementary Fig. S3 and Fig. 3B). To assess whether the same could be the case for the mutation in gntRl in the GNTR strain, we deleted this gene and found that the growth rate of the GNTR- $\Delta g n t R 1$ mutant on glucose was reduced by approximately 50\%, when compared to the GNTR strain (Fig. 3B). It has been shown previously that there are two functionally redundant GntR-type regulators encoded by gntRl and gntR2 in $C$. glutamicum, and a mutant lacking both $g n t R 1$ and $g n t R 2$ has a $60 \%$ reduction in glucose uptake and specific growth rate when compared to the WT (Frunzke et al., 2008). Since gntR2 is located in the CGP3 region, which is missing in the adapted strains (and in the $\Delta \mathrm{CGP} 3$ strain), the constructed GNTR- $\Delta g n t R 1$ mutant essentially would be identical to a $\Delta g n t R 1 \Delta g n t R 2$ mutant, which explains the similar phenotypes.

In the current study we used a minimal medium differing slightly from the more commonly used CGXII medium (Unthan et al., 2014), and for this reason we decided to test the strains in this medium as well. As expected, both the G15H and the DKI strain displayed comparable high specific growth rates, which were approximately 33\% higher than that of the WT (Fig. 3C).

\section{The gntR1 and ramA mutations improve the metabolic efficiency}

The above characterization revealed the involvement of the gntRl and $\operatorname{ramA}$ mutations in the faster growth phenotype, and this improvement was not caused by inactivation of either RamA or GntR1. To gain additional information, we decided to look into the global gene expression profile of the different strains. In Fig. 4A, we provided a simplified overview of the results obtained from an analysis of the transcriptome profiles for the WT, G15H, LUXR, and DKI strains. The hierarchical clustering results revealed that the 


\section{ACCEPTED MANUSCRIPT}

global gene expression profile of the adapted mutant G15H was similar to that of the double knock-in mutant DKI, however it was quite different from that of the WT strain. By applying KEGG enrichment analysis to interrogate the functions of differentially expressed genes (Fig. 4B) we could see that the genes that were differentially expressed in DKI and G15H were involved in cellular processes such as "microbial metabolism in diverse environments", carbon metabolism, glycolysis, and the pentose phosphate pathway (PPP), i.e. all growth-associated categories. The LUXR strain exhibited a less pronounced differential gene expression profile, and fewer cellular processes seemed to be affected when compared to that of G15H or DKI, which correlated well with the smaller effect on growth rate. Moreover, we compared the expression level of 33 genes involved in the central metabolism for the four tested strains (Fig. 4C) and found that most of the genes involved in glycolysis were significantly upregulated in LUXR and DKI, which could indicate that the mutated RamA has changed its function, to become a global up-regulator of glycolytic genes. The PPP genes were greatly upregulated in both DKI and G15H, which indicates an involvement of the mutated GntR. On the contrary, some TCA genes especially for $\operatorname{sucBD}$ were down-regulated in the fast growing cells. This may demonstrate the transcriptional control of TCA flux is low in C. glutamicum. In addition, the anaplerotic reactions encoded by $p p c$ were up-regulated, while the reverse reaction driven by $p c k$ was significantly down-regulated. According to a previous study, deletion of the prophage CGP3 region leads to a strong reduction in the expression of $p c k$ (Baumgart et al., 2013). More detailed information regarding the transcriptomics analysis is provided in Supplementary Data 2. 
A
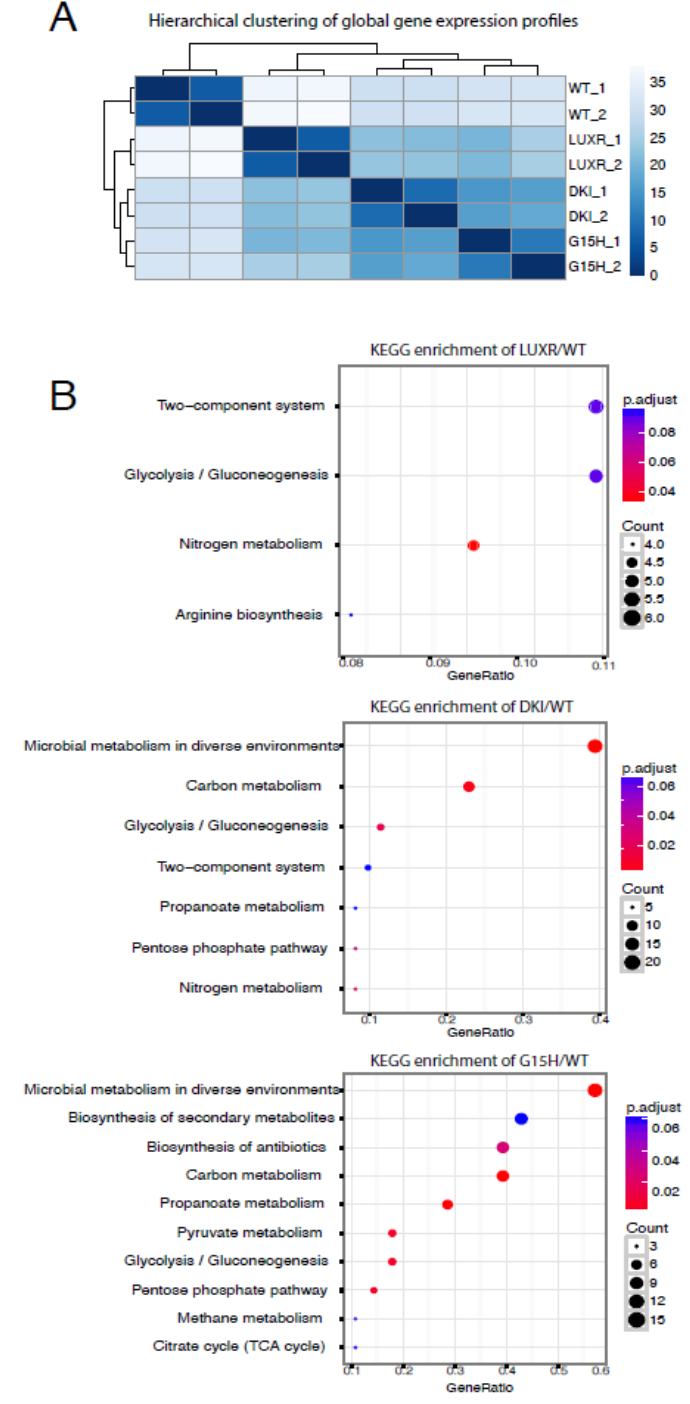

C
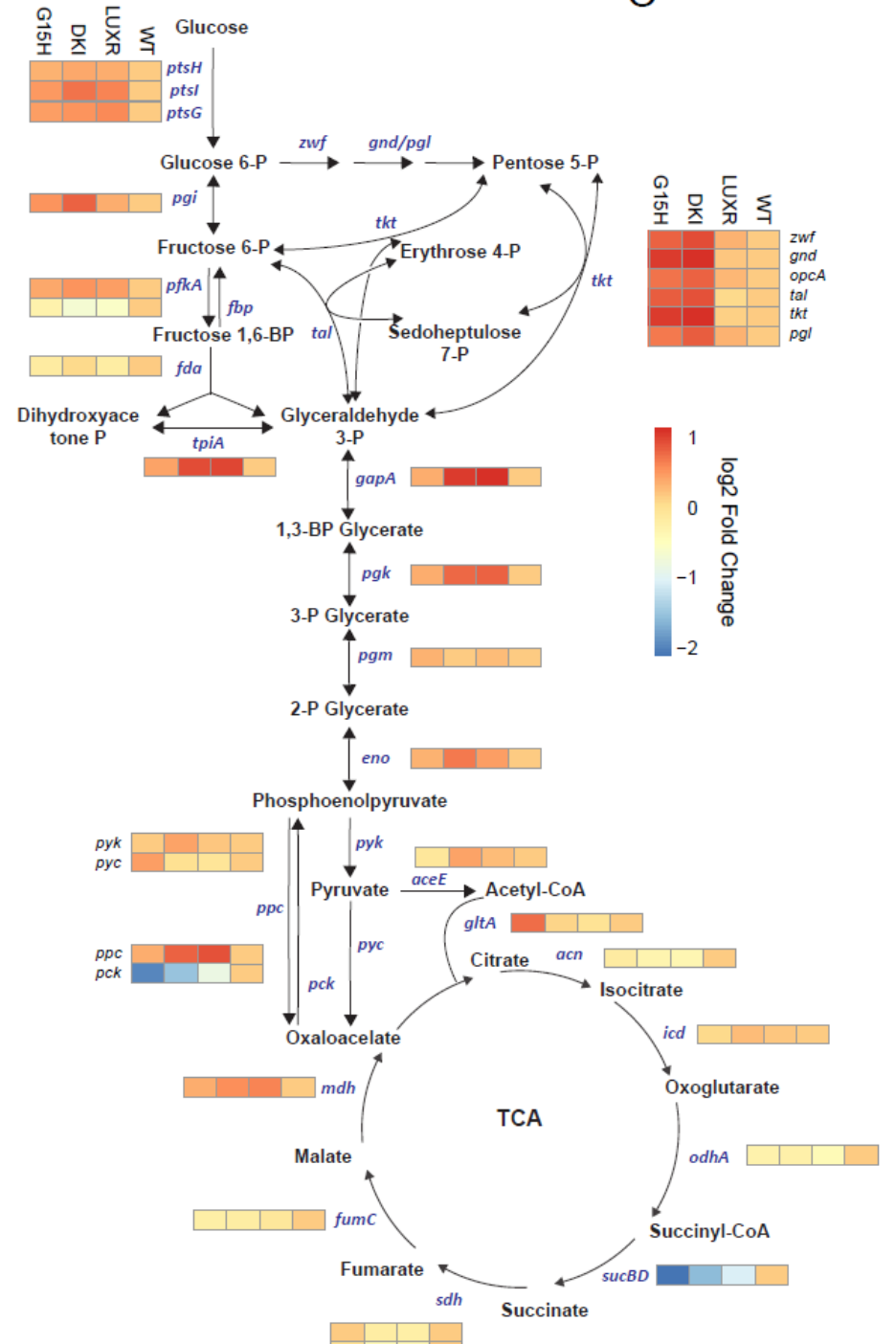

Fig. 4 Comparison of global gene expression and contextualization of gene expression data in the central metabolic network. (A) Hierarchical clustering of gene expression profiles for four tested strains after normalization using DEseq2. The transcriptomics data for each strain was obtained based on biological duplicates. (B) The genes that are differentially expressed among the reconstructed mutants and WT have been classified according to gene functional annotations by using KEGG enrichment analysis. The color of circles (from purple to red) represents the adjusted p-value from higher to lower, while the size of circles represents the number of qualified gene counts. (C) The expression of a series of genes involved in central metabolism is compared using a heat map. The strains from left to right are: G15H, DKI, LUXR, and WT respectively.

For the DKI and G15H strains the gntP (encoding gluconate permease) and gntK (encoding gluconate kinase) were strongly upregulated, to a level around 9- and 20-fold higher than in the WT strain, respectively 


\section{ACCEPTED MANUSCRIPT}

(Supplementary Data 2). This could indicate a potential role in the fast growth phenotype, however, deletion of either gene did not have any effect on growth (Supplementary Fig. S4).

In addition, the apparent growth rate limitation on glucose, for the wild-type, could be due to either a limitation in catabolism or anabolism. In the latter case it is commonly found that the intracellular energy charge, or more simply the ATP/ADP ratio, is high, whereas a limitation in catabolism can lead to a low ATP/ADP ratio (Koebmann et al., 2002b). We measured the intracellular ATP and ADP concentration of the WT, DKI, and G15H, and found that the ATP/ADP ratios for DKI and G15H were quite comparable, and around 25\% higher than that of the WT (Supplementary Fig. S5). This may indicate the presence of a limitation in catabolism in the WT strain, which the transcriptomics analysis also supported.

\section{${ }^{13} \mathrm{C}$ metabolic flux analysis}

Changes in transcription levels of genes involved in the central carbon metabolism can indicate metabolic shifts. To determine whether the high growth rate could be associated with changes in the metabolic flux redistribution, we performed ${ }^{13} \mathrm{C}-\mathrm{MFA}$ for the wild type and DKI strains. The results are shown in Fig. S6, where the fluxes shown have been normalized to 100 units of glucose uptake $\left(80 \% 1-{ }^{13} \mathrm{C}\right.$ and $\left.20 \% \mathrm{U}-{ }^{13} \mathrm{C}\right)$. We found that the relative metabolic fluxes in glycolysis and TCA cycle were remarkably similar for the two strains, but a small increase in the PPP flux was still found for the DKI strain. The raw measured and fitted labelling data can be found in Supplementary data 3. In absolute terms, as the glucose uptake rates were significantly elevated from $5.05 \mathrm{mmol} / \mathrm{g} \mathrm{DCW} / \mathrm{h}$ in the wild type to $6.92 \mathrm{mmol} / \mathrm{g} \mathrm{DCW} / \mathrm{h}$ in DKI, the overall metabolic rates were increased as shown in Fig. 5. These data show that, even though, the genes in glycolysis were broadly upregulated and the genes in TCA cycle were downregulated, this did not correspond to a significant change in the metabolic flux distribution. Our results are consistent with the recent findings of Long et al., for evolved E. coli strains, where the intracellular metabolic fluxes changed very little after ALE, although the growth rate had been increased by up to 1.6-fold (Long et al., 2017). They found that the faster growth of adaptively evolved strains was not due to an intracellular flux redistribution, but rather caused by increases in glucose uptake rate and an overall boosted central metabolism. In the 


\section{ACCEPTED MANUSCRIPT}

current flux analysis model, we considered the cellular composition to be stable so that it could not cover all potential adaptations in anabolic fluxes that might have occurred.

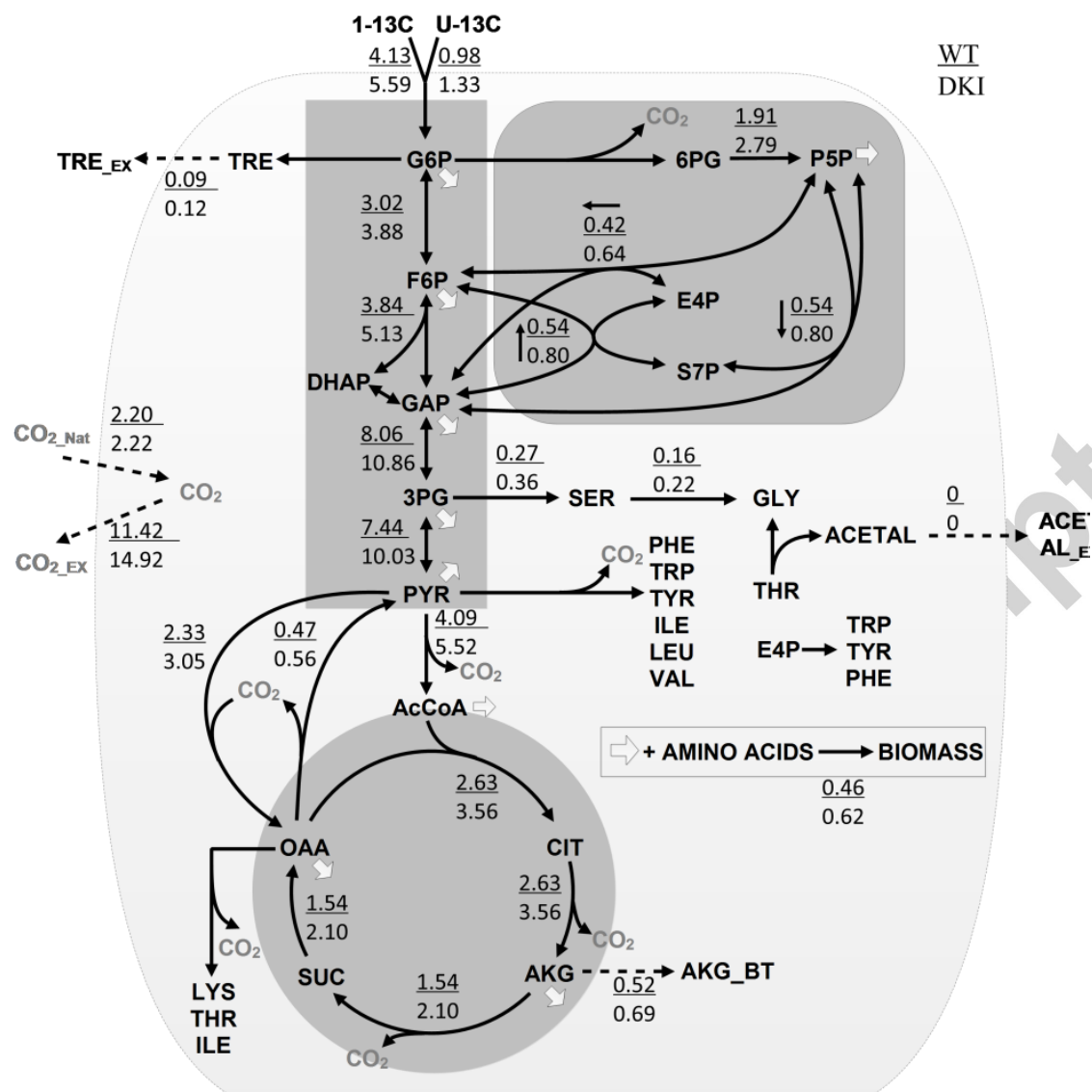

Fig. 5 Metabolic flux analysis of the central carbon metabolism in the wild type (WT, upper values) and DKI (lower values). All the fluxes are given in $\mathrm{mmol} / \mathrm{g} \mathrm{DCW} / \mathrm{h}$. The glucose uptake rates were $5.05 \mathrm{mmol} / \mathrm{g}$ DCW/h in the wild type and $6.92 \mathrm{mmol} / \mathrm{g}$ DCW/h in DKI, respectively. Abbreviates: $1-13 \mathrm{C}, 1-{ }^{13} \mathrm{C}$ glucose. U-13C, U- ${ }^{13}$ C glucose. G6P, glucose-6-phosphate. F6P, fructose-6-phosphate. GAP, glyceraldehyde 3-P. DHAP, dihydroxyacetone P. 3PG, 3-P glycerate. PYR, pyruvate. TRE, trehalose. 6PG, 6-phosphogluconate. P5P, pentose 5-P. E4P, erythrose 4-P. S7P, sedoheptulose 7-P. CIT, citrate. AKG, a-ketoglutarate. SUC, succinate. OAA, oxaloacetate.

The effect of the gntR1 and $\operatorname{ram} A$ mutations on growth is not restricted to glucose. 
We decided to investigate whether the beneficial effects on growth, conferred by the gntRI and ramA mutations, could be observed on other carbon sources besides glucose. As shown in Fig. 6, both the G15H and DKI strains were found to grow faster on sucrose and fructose, $\mathrm{G} 15 \mathrm{H}$ by approximately $20 \%\left(\mu_{\mathrm{G} 15 \mathrm{H}, \mathrm{Suc}}=\right.$ $\left.0.67 \pm 0.02 \mathrm{~h}^{-1} ; \mu_{\mathrm{G} 15 \mathrm{H}, \mathrm{Fru}}=0.61 \pm 0.01 \mathrm{~h}^{-1}\right)$ and the DKI strain by approximately $15 \%\left(\mu_{\mathrm{DKI}, \mathrm{Suc}}=0.64 \pm 0.01\right.$ $\left.\mathrm{h}^{-1} ; \mu_{\mathrm{DKI}, \text { Fru }}=0.59 \pm 0.01 \mathrm{~h}^{-1}\right)$ when compared to the WT $\left(\mu_{\mathrm{WT}, \text { Suc }}=0.56 \pm 0.02 \mathrm{~h}^{-1} ; \mu_{\mathrm{WT}, \text { Fru }}=0.51 \pm 0.02 \mathrm{~h}^{-1}\right)$. Similar to the phenotype on glucose, here we also tested the growth rate of the $\Delta$ CGP3 strain on sucrose and fructose, however, the strain grew similar to that of the WT (Figure not shown). In addition, the plasmid pXYL, a derivative of pECX99E containing $x y l A$ and $x y l B$ gene from Lactococcus latis KF147 for xylose metabolism, was transformed into the WT and DKI strain as well. The latter strain $\left(\mu_{\mathrm{DKI}-\mathrm{pXYL}, \mathrm{Xyl}}=0.18 \pm\right.$ $\left.0.01 \mathrm{~h}^{-1}\right)$ also exhibited a $37 \%$ higher growth rate compared to the WT-pXYL strain $\left(\mu_{\mathrm{WT}-\mathrm{pXYL}, \mathrm{Xyl}}=0.13 \pm\right.$ $0.02 \mathrm{~h}^{-1}$ ) when growing on xylose as the sole carbon source. In contrast, on the gluconeogenic carbon sources lactate and acetate, the three strains performed very similarly (Fig. 6). Also, the stimulatory effect of the mutations could only be seen on minimal medium, and not when the strains were grown in rich BHI medium.

Sucrose

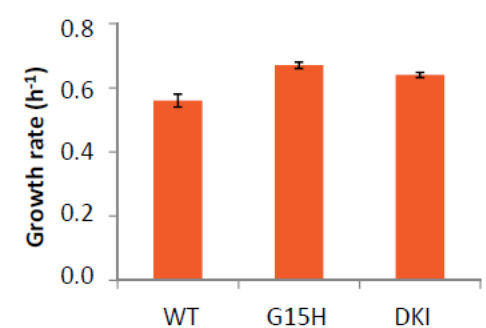

Acetate

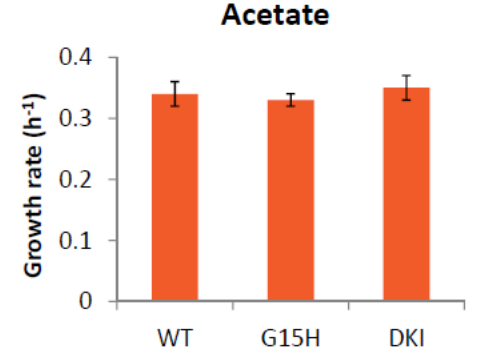

Fructose

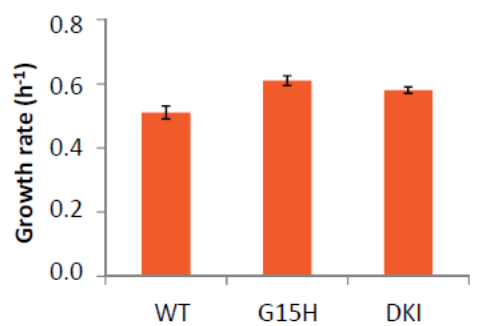

Lactate

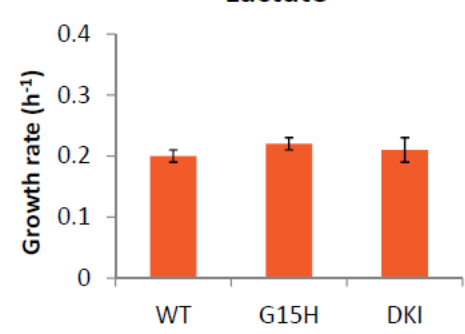

Xylose

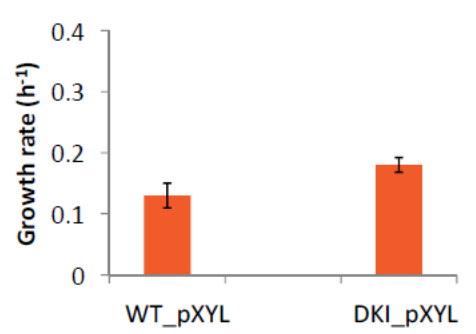

BHI

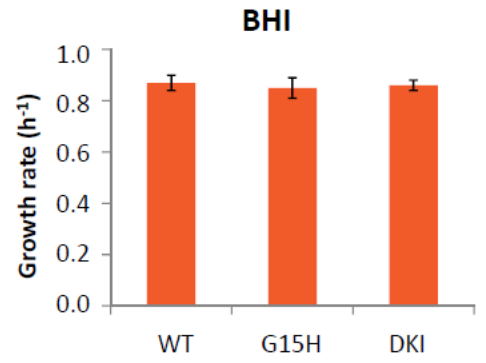




\section{ACCEPTED MANUSCRIPT}

Fig. 6 Comparison of growth rates for the WT, G15H, and DKI strain growing on different carbon sources. The concentration of sucrose and fructose was $10 \mathrm{~g} / \mathrm{L}$, whereas the concentration of lactate and acetate was $150 \mathrm{mM}$. BHI (Brain heart infusion, Sigma) is a rich medium supporting fast growth. The specific growth rates are calculated based on data from three biological replicates.

\section{The gntR1 and ramA mutations enhance glycolytic capacity under anaerobic conditions}

Several interesting studies have shown the potential of using C. glutamicum as a whole cell catalyst under anaerobic conditions where the cells are unable to grow (Inui et al., 2004; Michel et al., 2015). Both the ramA and the gntRl mutations had a positive effect on the glycolytic flux when the cells were growing, and for this reason, we decided to investigate whether the mutations also could have an effect under anaerobic conditions. The different strains were first grown in rich medium, harvested and then re-suspended to an $\mathrm{OD}_{600} \approx 20$ in minimal medium with $2 \%$ glucose, after which glucose consumption and lactate production rates were compared (at $30^{\circ} \mathrm{C}$ ). Since lactate is formed under these conditions, the medium was supplemented with $200 \mathrm{mM}$ MOPS serving as a pH buffer. As shown in Fig. 7, G15H and DKI consumed glucose at comparable rates $\left(\mathrm{q}_{\mathrm{G} 15 \mathrm{H}, \mathrm{glu}}=2.75 \pm 0.18 \mathrm{mmol} / \mathrm{g} \cdot \mathrm{DCW} / \mathrm{h} ; \mathrm{q}_{\mathrm{DKI}, \mathrm{glu}}=2.71 \pm 0.22 \mathrm{mmol} / \mathrm{g} \cdot \mathrm{DCW} / \mathrm{h}\right)$ and lactate formation rates were also quite similar for the two strains $\left(\mathrm{q}_{\mathrm{G} 15 \mathrm{H}, \mathrm{lac}}=3.67 \pm 0.27 \mathrm{mmol} / \mathrm{g} \cdot \mathrm{DCW} / \mathrm{h}\right.$; $\left.\mathrm{q}_{\mathrm{DKI}, \mathrm{lac}}=3.62 \pm 0.22 \mathrm{mmol} / \mathrm{g} \cdot \mathrm{DCW} / \mathrm{h}\right)$. When compared to the WT these fluxes were approximately $30 \%$ higher than that of the WT $\left(\mathrm{q}_{\mathrm{wT}, \mathrm{glu}}=2.09 \pm 0.16 \mathrm{mmol} / \mathrm{g} \cdot \mathrm{DCW} / \mathrm{h} ; \mathrm{q}_{\mathrm{WT}, \mathrm{lac}}=2.81 \pm 0.23 \mathrm{mmol} / \mathrm{g} \cdot \mathrm{DCW} / \mathrm{h}\right)$. In a previous study it was shown that, by over-expressing several glycolytic genes ( $p g i, p f k, g a p A$, and $p y k)$, the anaerobic glucose metabolism and alanine production (Yamamoto et al., 2012) could be improved. Our results reveal a more simple way to achieve the similar effect, i.e. introducing mutations in gntRI and $\mathrm{ramA}$. However, the previous study was unable to stimulate the glycolytic flux in growing cells as was accomplished in our study. 


\section{ACCEPTED MANUSCRIPT}
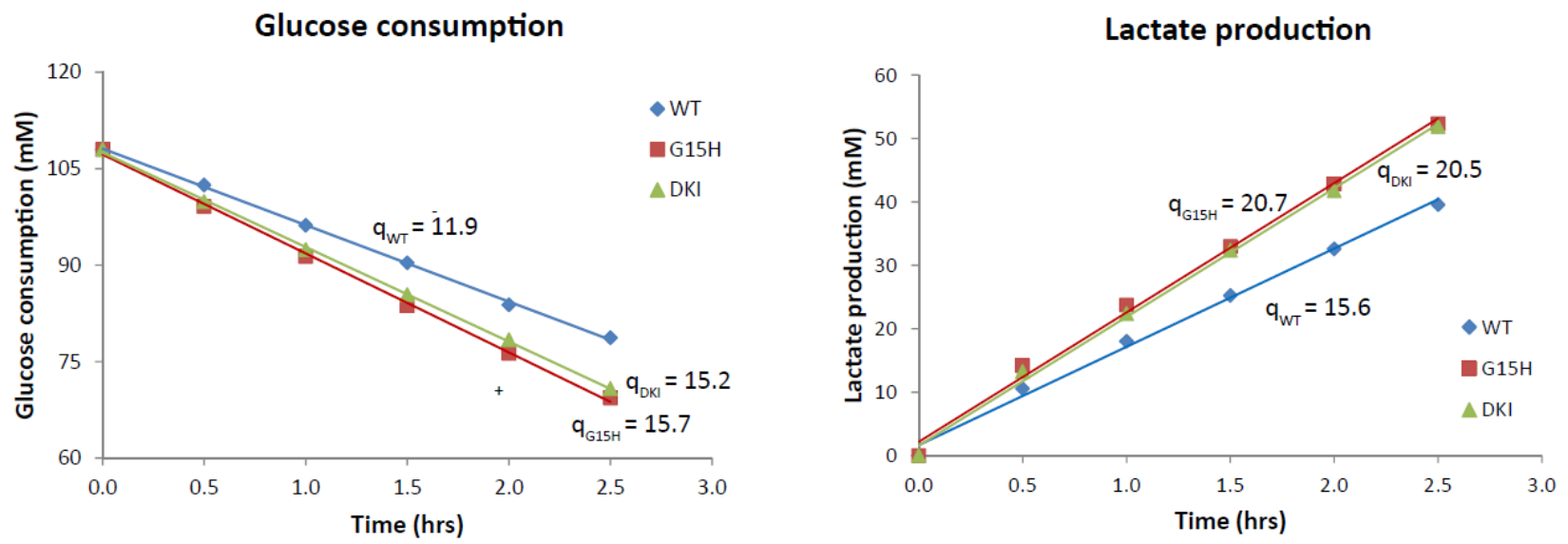

Fig. 7 Glucose consumption and lactate formation rates for WT, G15H, and DKI under anaerobic conditions. Cells grown in rich BHI medium were re-suspended in minimal medium with $2 \%$ glucose and $200 \mathrm{mM}$ MOPS buffer to an $\mathrm{OD}_{600} \approx 20$. Data from one representative out of three similar biological replicates is shown.

\section{The gntR1 and ramA mutations enhance the lysine yield and productivity}

Finally, we tested the effects of gntRl an $\operatorname{ramA}$ mutations on lysine production. The two mutations were introduced into a lysine-producing strain (LysC), containing a mutation in lys $C$ conferring feedbackresistance ( Thr-311->Ile in lysC gene, Ohnishi et al., 2002). As shown in Fig. 8, we compared the performance of LysC and DKI_LysC strain in MM with $0.5 \%$ glucose, and observed that the specific growth indeed was increased from $0.43 \mathrm{~h}^{-1}$ (LysC) to $0.59 \mathrm{~h}^{-1}$ (DKI_LysC), i.e. the same effect as observed for the WT. More interestingly, the lysine titer was also increased 32\% for the DKI_LysC strain. Because of the mutations, the entire fermentation process could be shortened from 11 hrs to 9 hrs (Fig. 8), which corresponds to an average increase in volumetric lysine productivity of $100 \%$. The effect that these two mutations have on the performance of high-yield, high titer industrial lysine-producing strains remains to be seen. 


\section{ACCEPTED MANUSCRIPT}

A

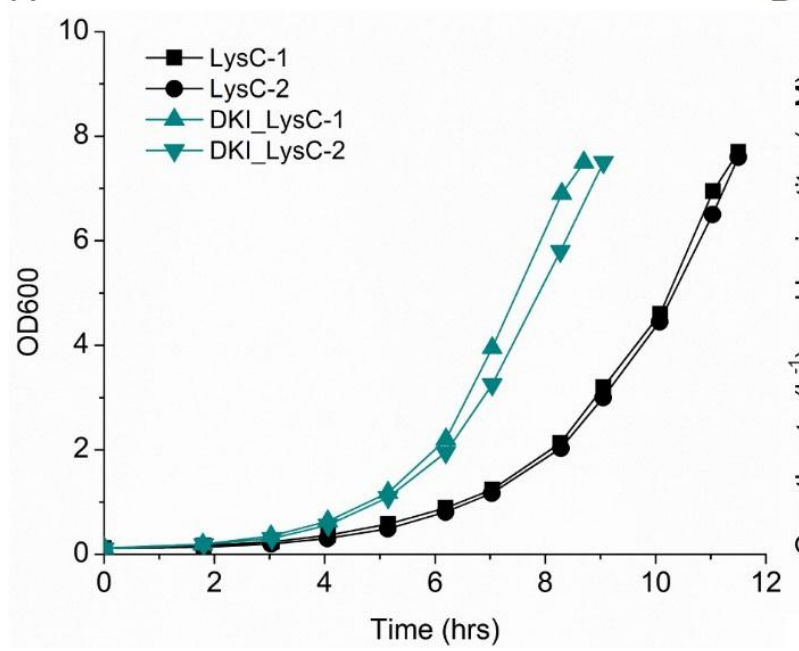

$B$

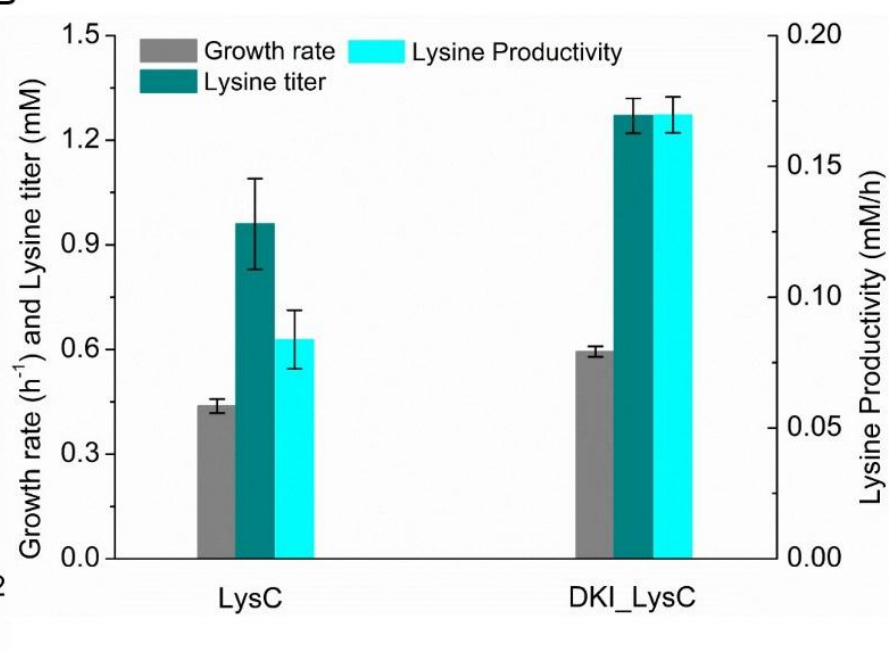

Fig. 8 Characterization for the LysC and DKI_LysC strain. Cells were cultivated in the minimum medium with $5 \mathrm{~g} / \mathrm{L}$ glucose. A. the growth. B. the specific growth rate, lysine titer and lysine productivity. The growth characterization was performed by biological duplicates.

\section{Discussion}

As mentioned above, the specific growth rate is an important parameter for microorganisms with industrial relevance, such as C. glutamicum. C. glutamicum grows well in cheap defined media, albeit with a somewhat low specific growth rate $\left(0.45 \mathrm{~h}^{-1}\right)$ and few studies have focused on what limits the specific growth rate for this particular organism (Keilhauer et al., 1993; Liebl et al., 1989). Recently, Noack and his colleagues reported a high specific growth rate of $0.61 \mathrm{~h}^{-1}$ for $C$. glutamicum growing in a defined minimal medium containing glucose (Unthan et al., 2014) and protocatechuic acid (PCA). The reason for the fast growth was found to be simultaneous metabolism of glucose and PCA, which enter metabolism at different points. Where glucose is metabolized via glycolysis, PCA in few steps is converted into succinyl-CoA and acetylCoA that directly enter the TCA cycle. For industrial applications such co-feeding approaches are only useful when the substrates are cheap, and using PCA is not a viable option due to its high cost. It is therefore relevant to investigate whether the specific growth rate could be increased by other means. Through longterm ALE, we successfully isolated several mutants which grew faster $\left(\mu_{\max } \approx 0.64 \mathrm{~h}^{-1}\right)$ than the WT and we 


\section{ACCEPTED MANUSCRIPT}

identified the mutations responsible for most of the growth improvement and demonstrated that these SNPs can be stably inherited (Fig. 2).

One important mutation was located in gntRI (cg2783), encoding GntR1, which previously has been identified as a transcriptional regulator of gluconate catabolism together with GntR2 encoded by gntR2 (cg1935) (Frunzke et al., 2008). These two regulators have been proven to be functionally redundant. It has been demonstrated that the gluconate permease $($ gnt $P)$, gluconate kinase $($ gnt $K)$, gluconate 6-phosphat dehydrogenase ( $g n d$ ) and genes in the PPP are all strongly repressed by GntR1 and GntR2, while the glucose PTS (ptsG) is activated by them. A mutant lacking both regulators metabolizes glucose $60 \%$ slower, and therefore also grows quite slowly. However, in this study, we found that gntR2 had been lost along with the prophage region (CGP3) in the fast growing adapted mutants, and that gntRl had been mutated (gntRlE70K), which resulted in an increased expression of gntP (10.5-fold) and gntK (42.2-fold). Frunzke et al., 2008 characterized a $\Delta g n t R 1 \Delta g n t R 2$ mutant (Frunzke et al., 2008) and observed the same effect on expression of these two genes. However, in contrast to what we found for the deletion strain, neither the expression of $p t s G$ nor cell growth rate on glucose had been reduced in the gntRl-E70K strain, which indicated that the E70K mutation did not inactivate gntRl completely. Since the binding motif of gntP (TTTGATCATACTAAT) and gntK (TATGATAGTACCAAT) only shared little similarity with that of ptsG (AAAAGTATTACCTTT), we hypothesize that the effects from the E70K mutation on expression of these genes might be different.

The RamA (cg2831) protein is a LuxR-type global transcriptional regulator in C. glutamicum and it controls expression of genes involved in sugar uptake, glycolysis, gluconeogenesis, acetate, and several other metabolic pathways (Auchter et al., 2011). It was first identified as a transcriptional activator of genes involved in acetate metabolism by its binding to the promoter region of pta-ack, and its inactivation was found to completely abolish growth on acetate (Cramer A et al., 2006). Later it was shown that RamA could serve as a repressor of the glycolytic gene gapA (Toyoda et al., 2009) and as a positive regulator of the TCA cycle genes $s d h C A B$ (succinate dehydrogenase operon) and acn (aconitase) (Bussmann et al., 2009; Teramoto et al., 2011). In total 55 genes appear to be regulated by RamA, of which 45 have been verified experimentally and the remaining candidates have been predicted to be regulated as shown in CoryneRegNet 


\section{ACCEPTED MANUSCRIPT}

(http://www.coryneregnet.de) (Pauling et al., 2012). However, despite accumulating knowledge regarding the regulatory roles of RamA, there have been no reports describing beneficial effects on central metabolism from mutations in ramA. In this study we found such a mutation, which did not result in impairment of acetate metabolism. The transcriptomics data on glucose indicated that the glucose PTS and glycolytic genes were up-regulated to some extent, which correlated nicely with the increased glucose uptake rate and growth rate, but a specific bottleneck responsible for the slower growth of the wild-type could not be pinpointed. Indeed, growth may be regulated in a more complicated manner, and simple overexpression of a "ratelimiting" step might not be sufficient to overcome such regulatory mechanisms. We found that the expression of most TCA cycle genes were slightly decreased except for the succinyl-CoA synthase genes $s u c B D$, however, no effects were observed on the TCA cycle flux (Fig. S6).

When this article was under review, a study describing a $C$. glutamicum mutant with a $28 \%$ improvement in specific growth rate was published (Pfeifer et al. 2017). Although a similar approach was used to obtain this mutant, fast growth was found to be due to different mutations, e.g. in genes encoding pyruvate kinase and 1phosphofructokinase $(f r u K)$. The latter mutation is interesting as we previously have found that a fruK and ptsF double mutant is able to grow $10 \%$ faster in glucose minimal medium (Wang et al., 2016). In the study by Pfeifer et al. growth characterization was carried out in a Biolector, i.e. automated equipment, using a $750 \mu \mathrm{l}$ fermentation volume. Curiously for the same wild-type strain used in our study, and using the same medium CGXII, they obtained a specific growth rate that was $16 \%$ higher than what we achieved using standard shake flasks. It is possible that the small fermentation volume allowed for a more efficient aeration, and that this could somehow explain the effect. Another difference between these two studies is that the large truncations found in the genome of our evolved strains (Fig. S1) did not appear in their evolved strains. This may be due to the different evolution method or medium (MM or CGXII) used. However, both studies clearly demonstrate that there is space to improve the growth fitness of C. glutamicum. In this study we also characterized growth on other carbon sources in addition to glucose. Interestingly, when grown on sucrose and fructose, the G15H and DKI strain also displayed a 15\% 20\% increase in specific growth rate when compared to the WT strain. This indicates that the glycolytic capacity, in general, has been

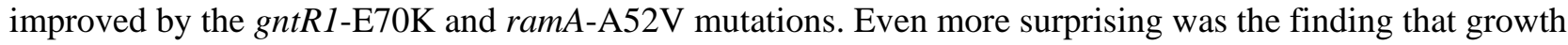


on xylose could be improved (a strain containing a plasmid expressing xylAB from L. lactis) by the gntRI and $\operatorname{ram} A$ mutations, despite the fact that the strain without the mutations grew very slowly, and had a very low glycolytic flux indicating that glycolytic capacity should not be an issue. In addition, it has been shown that the glycolytic flux in non-growing cells is far below that of growing cells, and that this can be explained by the reduced ATP demand in non-growing cells (Koebmann et al., 2002a). Surprisingly Yamamoto et al., 2012 were able to increase the glycolytic flux in non-growing cells by overexpressing various glycolytic genes, which seems counter-intuitive as a build-up of ATP would be expected to slow down glycolysis. We decided to examine whether our faster growing strains also performed better under such conditions, and indeed we observed an increased glucose uptake and lactate production for the non-growing cells.

To elucidate how the mutations in gntRl and $\operatorname{ramA}$ change their function is next in the pipeline, and for this purpose a reliable protein structure model could be useful for predicting the potentially altered regulation or activation site by the single amino acid variation.

\section{Conclusion}

Using adaptive laboratory evolution we have obtained a mutant of $C$. glutamicum with the hitherto fastest growth on glucose minimal medium, and have found the main reason for the fast growth to be two mutated transcriptional regulators $g n t R 1-\mathrm{E} 70 \mathrm{~K}$ and $\mathrm{ramA}-\mathrm{A} 52 \mathrm{~V}$. The beneficial effect on growth of these mutations is not restricted to glucose alone, and growth on the important industrially relevant carbohydrates sucrose, fructose and xylose was stimulated as well. We thus demonstrate that it is possible to achieve remarkable improvements in performance of this highly important industrial workhorse by minimal manipulation, which is important to avoid unnecessary metabolic perturbations and to maximize the efficiency of C. glutamicum based cell factories.

\section{Acknowledgments}

This work was supported by the Bio-Value Strategic Platform for Innovation and Research which is cofunded by The Danish Council for Strategic Research and The Danish Council for Technology and Innovation, case no: 0603-00522B. We would like to appreciate the strains (MB001 and $\triangle \mathrm{CGP} 3$ ) donated by 
Prof. Julia Frunzke and Prof. Stephan Noack (IBG-1, Forschungszentrum Jülich GmbH, Germany). We appreciate the suggestion and help from Prof. Birgitta Ebert and Dr. Ulf Liebal (RWTH Aachen University, Germany). We would also like to acknowledge all our co-workers especially Jun Chen, Mike Vestergaard, Jing Shen, Tine Suhr.

\section{Author contributions}

P.R.J. and C.S. conceived the project. P.R.J., C.S., and Z.W. designed the research and coordinated the project. Z.W., J. L., and L. C. performed the experimental works and data analysis with the help from A. Z. Z.W prepared the manuscript with the help from J.L, P.R.J, and C.S.

\section{Reference}

Albert, T.J., Dailidiene, D., Dailide, G., Norton, J.E., Kalia, A., Richmond, T. a, Molla, M., Singh, J., Green, R.D., Berg, D.E., 2005. Mutation discovery in bacterial genomes: metronidazole resistance in Helicobacter pylori. Nat. Methods 2, 951-953. doi:10.1038/nmeth805

Anders, S., Pyl, P.T., Huber, W., 2015. HTSeq-A Python framework to work with high-throughput sequencing data. Bioinformatics 31, 166-169. doi:10.1093/bioinformatics/btu638

Auchter, M., Cramer, A., Hüser, A., Rückert, C., Emer, D., Schwarz, P., Arndt, A., Lange, C., Kalinowski, J., Wendisch, V.F., Eikmanns, B.J., 2011. RamA and RamB are global transcriptional regulators in Corynebacterium glutamicum and control genes for enzymes of the central metabolism. J. Biotechnol. 154, 126-139. doi:10.1016/j.jbiotec.2010.07.001

Bailey, J.E., Sburlati, A., Hatzimanikatis, V., Lee, K., Renner, W.A., Tsai, P.S., 1996. Inverse metabolic engineering: A strategy for directed genetic engineering of useful phenotypes. Biotechnol. Bioeng. 52, 109-121.

Barrick, J.E., Yu, D.S., Yoon, S.H., Jeong, H., Oh, T.K., Schneider, D., Lenski, R.E., Kim, J.F., 2009. Genome evolution and adaptation in a long-term experiment with Escherichia coli. Nature 461, 1243-1247. doi:10.1038/nature08480

Bäumchen, C., Knoll, A., Husemann, B., Seletzky, J., Maier, B., Dietrich, C., Amoabediny, G., Büchs, J., 2007. Effect of elevated dissolved carbon dioxide concentrations on growth of Corynebacterium glutamicum on d-glucose and l-lactate. J. Biotechnol. 128, 868-874. doi:10.1016/j.jbiotec.2007.01.001

Baumgart, M., Unthan, S., Rückert, C., Sivalingam, J., Grünberger, A., Kalinowski, J., Bott, M., Noack, S., Frunzke, J., 2013. Construction of a Prophage-Free Variant of Corynebacterium 
glutamicum ATCC 13032 for Use as a Platform Strain for Basic Research and Industrial Biotechnology. Appl. Environ. Microbiol. 79, 6006-6015. doi:10.1128/AEM.01634-13

Becker, J., Wittmann, C., 2015. Advanced Biotechnology: Metabolically Engineered Cells for the Bio-Based Production of Chemicals and Fuels, Materials, and Health-Care Products. Angew. Chemie Int. Ed. 54, 3328-3350. doi:10.1002/anie.201409033

Becker, J., Wittmann, C., 2012. Bio-based production of chemicals, materials and fuels Corynebacterium glutamicum as versatile cell factory. Curr. Opin. Biotechnol. 23, 631-40. doi:10.1016/j.copbio.2011.11.012

Becker, J., Zelder, O., Häfner, S., Schröder, H., Wittmann, C., 2011. From zero to hero--designbased systems metabolic engineering of Corynebacterium glutamicum for L-lysine production. Metab. Eng. 13, 159-168. doi:10.1016/j.ymben.2011.01.003

Bussmann, M., Emer, D., Hasenbein, S., Degraf, S., Eikmanns, B.J., Bott, M., 2009. Transcriptional control of the succinate dehydrogenase operon sdhCAB of Corynebacterium glutamicum by the cAMP-dependent regulator GlxR and the LuxR-type regulator RamA. J. Biotechnol. 143, 173-182. doi:10.1016/j.jbiotec.2009.06.025

Caspeta, L., Chen, Y., Ghiaci, P., Feizi, a., Buskov, S., Hallstrom, B.M., Petranovic, D., Nielsen, J., 2014. Altered sterol composition renders yeast thermotolerant. Science (80-. ). 346, 75-78. doi:10.1126/science. 1258137

Chan, S.H.J., Nørregaard, L., Solem, C., Jensen, P.R., 2014. Acetate kinase isozymes confer robustness in acetate metabolism. PLoS One 9, e92256. doi:10.1371/journal.pone.0092256

Cheng, K.-K., Lee, B.-S., Masuda, T., Ito, T., Ikeda, K., Hirayama, A., Deng, L., Dong, J., Shimizu, K., Soga, T., Tomita, M., Palsson, B.O., Robert, M., 2014. Global metabolic network reorganization by adaptive mutations allows fast growth of Escherichia coli on glycerol. Nat. Commun. 5, 3233. doi:10.1038/ncomms4233

Cramer A, Gerstmeir R, Schaffer S, Bott M, E.B., 2006. Identification of RamA, a novel LuxR-type transcriptional regulator of genes involved in acetate metabolism of Corynebacterium glutamicum. J. Bacteriol. 188, 2554-2567. doi:10.1128/JB.186.9.2798

DePristo, M. a., Banks, E., Poplin, R.E., Garimella, K. V., Maguire, J.R., Hartl, C., Philippakis, a. a., del Angel, G., Rivas, M. a, Hanna, M., McKenna, A., Fennell, T.J., Kernytsky, a. M., Sivachenko, a. Y., Cibulskis, K., Gabriel, S.B., Altshuler, D., Daly, M.J., 2011. A framework for variation discovery and genotyping using next- generation DNA sequencing data. Nat Genet 43, 491-498. doi:10.1038/ng.806.A

Dettman, J.R., Rodrigue, N., Melnyk, A.H., Wong, A., Bailey, S.F., Kassen, R., 2012. Evolutionary insight from whole-genome sequencing of experimentally evolved microbes. Mol. Ecol. 21, 2058-2077. doi:10.1111/j.1365-294X.2012.05484.x

Engels, V., Wendisch, V.F., 2007. The DeoR-type regulator SugR represses expression of ptsG in Corynebacterium glutamicum. J. Bacteriol. 189, 2955-2966. doi:10.1128/JB.01596-06

Feist, A.M., Zielinski, D.C., Orth, J.D., Schellenberger, J., Herrgard, M.J., Palsson, B.O., 2010. Model-driven evaluation of the production potential for growth-coupled products of 
Escherichia coli. Metab. Eng. 12, 173-186. doi:10.1016/j.ymben.2009.10.003

Frunzke, J., Engels, V., Hasenbein, S., Gätgens, C., Bott, M., 2008. Co-ordinated regulation of gluconate catabolism and glucose uptake in Corynebacterium glutamicum by two functionally equivalent transcriptional regulators, GntR1 and GntR2. Mol. Microbiol. 67, 305-322. doi:10.1111/j.1365-2958.2007.06020.x

Gibson, D.G., Young, L., Chuang, R.-Y., Venter, J.C., Hutchison, C. a, Smith, H.O., Iii, C.A.H., America, N., 2009. Enzymatic assembly of DNA molecules up to several hundred kilobases. Nat. Methods 6, 343-5. doi:10.1038/nmeth.1318

Grünberger, A., van Ooyen, J., Paczia, N., Rohe, P., Schiendzielorz, G., Eggeling, L., Wiechert, W., Kohlheyer, D., Noack, S., 2013. Beyond growth rate 0.6: Corynebacterium glutamicum cultivated in highly diluted environments. Biotechnol. Bioeng. 110, 220-8. doi:10.1002/bit.24616

Herring, C.D., Raghunathan, A., Honisch, C., Patel, T., Applebee, M.K., Joyce, A.R., Albert, T.J., Blattner, F.R., van den Boom, D., Cantor, C.R., Palsson, B.Ø., 2006. Comparative genome sequencing of Escherichia coli allows observation of bacterial evolution on a laboratory timescale. Nat. Genet. 38, 1406-1412. doi:10.1038/ng1906

Hong, K.-K., Vongsangnak, W., Vemuri, G.N., Nielsen, J., 2011. Unravelling evolutionary strategies of yeast for improving galactose utilization through integrated systems level analysis. Proc. Natl. Acad. Sci. U. S. A. 108, 12179-12184. doi:10.1073/pnas.1103219108

Ibarra, R.U., Edwards, J.S., Palsson, B.O., 2002. Escherichia coli K-12 undergoes adaptive evolution to achieve in silico predicted optimal growth. Nature 420, 186-189. doi:10.1038/nature01149

Ikeda, M., 2012. Sugar transport systems in Corynebacterium glutamicum: features and applications to strain development. Appl. Microbiol. Biotechnol. 96, 1191-200. doi:10.1007/s00253-0124488-z

Inui, M., Murakami, S., Okino, S., Kawaguchi, H., Vertès, A.A., Yukawa, H., 2004. Metabolic analysis of Corynebacterium glutamicum during lactate and succinate productions under oxygen deprivation conditions. J. Mol. Microbiol. Biotechnol. 7, 182-196. doi:10.1159/000079827

Jakob, K., Satorhelyi, P., Lange, C., Wendisch, V.F., Silakowski, B., Scherer, S., Neuhaus, K., 2007. Gene expression analysis of Corynebacterium glutamicum subjected to long-term lactic acid adaptation. J. Bacteriol. 189, 5582-5590. doi:10.1128/JB.00082-07

James Winkler, Luis H. Reyes, and K.C.K., 2013. Adaptive Laboratory Evolution for Strain Engineering, in: Alper, H.S. (Ed.), Systems Metabolic Engineering: Methods and Protocols. Springer Protocols, pp. 211-22. doi:10.1007/978-1-62703-299-5

Jin, Y., Alper, H., Yang, Y.-T., Stephanopoulos, G., 2005. Improvement of xylose uptake and ethanol production in recombinant Saccharomyces cerevisiae through an inverse metabolic engineering approach. Appl. Environ. Microbiol. 71, 8249-8256.

doi:10.1128/AEM.71.12.8249 
Kalinowski, J., Bathe, B., Bartels, D., Bischoff, N., Bott, M., Burkovski, A., Dusch, N., Eggeling, L., Eikmanns, B.J., Gaigalat, L., Goesmann, A., Hartmann, M., Huthmacher, K., Krämer, R., Linke, B., McHardy, A.C., Meyer, F., Möckel, B., Pfefferle, W., Pühler, A., Rey, D. a, Rückert, C., Rupp, O., Sahm, H., Wendisch, V.F., Wiegräbe, I., Tauch, A., 2003. The complete Corynebacterium glutamicum ATCC 13032 genome sequence and its impact on the production of l-aspartate-derived amino acids and vitamins. J. Biotechnol. 104, 5-25. doi:10.1016/S01681656(03)00154-8

Katsumata, R., Ozaki, A., Oka, T., Furuya, A., 1984. Protoplast Transformation of GlutamateProducing Bacteria with Plasmid DNA. J. Bacteriol. 159, 306-311.

Keilhauer, C., Eggeling, L., Sahm, H., 1993. Isoleucine synthesis in Corynebacterium glutamicum: Molecular analysis of the ilvB-ilvN-ilvC operon. J. Bacteriol. 175, 5595-5603.

Kim, D., Langmead, B., Salzberg, S.L., 2015. HISAT: a fast spliced aligner with low memory requirements. Nat. Methods 12, 357-60. doi:10.1038/nmeth.3317

Kim, W.S., Park, J.H., Ren, J., Su, P., Dunn, N.W., 2001. Survival Response and Rearrangement of Plasmid DNA of Lactococcus lactis during Long-Term Starvation. Appl. Environ. Microbiol. 67, 4594-4602. doi:10.1128/AEM.67.10.4594-4602.2001

Kind, S., Jeong, W.K., Schröder, H., Wittmann, C., 2010. Systems-wide metabolic pathway engineering in Corynebacterium glutamicum for bio-based production of diaminopentane. Metab. Eng. 12, 341-51. doi:10.1016/j.ymben.2010.03.005

Koebmann, B.J., Solem, C., Pedersen, M.B., Nilsson, D., Jensen, P.R., 2002a. Expression of Genes Encoding F 1 -ATPase Results in Uncoupling of Glycolysis from Biomass Production in Lactococcus lactis. Appl. Environ. Microbiol. 68, 4274-4282. doi:10.1128/AEM.68.9.4274

Koebmann, B.J., Westerhoff, H. V, Snoep, J.L., Nilsson, D., Jensen, P.R., 2002b. The Glycolytic Flux in Escherichia coli Is Controlled by the Demand for ATP. J. Bacteriol. 184, 3909-3916. doi:10.1128/JB.184.14.3909

Kromer, J.O., Sorgenfrei, O., Klopprogge, K., Heinzle, E., Wittmann, C., 2004. In-depth profiling of lysine-producing Corynebacterium glutamicum by combined analysis of the transcriptome, metabolome and fluxome. J. Bacteriol. 186, 1769-1784.

Langmead, B., Salzberg, S.L., 2012. Fast gapped-read alignment with Bowtie 2. Nat. Methods 9, 357-359. doi:10.1038/nmeth.1923

Lee, S.Y., Kim, H.U., 2015. Systems strategies for developing industrial microbial strains. Nat. Biotechnol. 33, 1061-1072. doi:10.1038/nbt.3365

Liebl, W., Klamer, R., Schleifer, K., 1989. Requirement of chelating compounds for the growth of Corynebacterium ylutamicum in synthetic media. Appl. Microbiol. Biotechnol. 32, 205-210.

Liu, J., Chan, S.H.J., Brock-Nannestad, T., Chen, J., Lee, S.Y., Solem, C., Jensen, P.R., 2016a. Combining metabolic engineering and biocompatible chemistry for high-yield production of homo-diacetyl and homo-(S,S)-2,3-butanediol. Metab. Eng. 36, 57-67.

Liu, J., Kandasamy, V., Würtz, A., Jensen, P.R., Solem, C., 2016b. Stimulation of acetoin production in metabolically engineered Lactococcus lactis by increasing ATP demand. Appl. 
Microbiol. Biotechnol. 1-9. doi:10.1007/s00253-016-7687-1

Liu, J., Solem, C., Jensen, P.R., 2016c. Integrating biocompatible chemistry and manipulating cofactor partitioning in metabolically engineered Lactococcus lactis for fermentative production of (3S)-acetoin. Biotechnol. Bioeng.113, 2744-48.

Liu, J., Wang, Z., Kandasamy, V., Lee, S.Y., Solem, C., Jensen, P.R., 2017. Harnessing the respiration machinery for high-yield production of chemicals in metabolically engineered Lactococcus lactis. Metab. Eng. 44, 22-29.

Long, C.P., Gonzalez, J.E., Feist, A.M., Palsson, B.O., Antoniewicz, M.R., 2017. Fast growth phenotype of E. coli K-12 from adaptive laboratory evolution does not require intracellular flux rewiring. Metab. Eng. doi:10.1016/j.ymben.2017.09.012

Love, M.I., Huber, W., Anders, S., 2014. Moderated estimation of fold change and dispersion for RNA-seq data with DESeq2. Genome Biol. 15, 550. doi:10.1186/s13059-014-0550-8

Mahr, R., Gätgens, C., Gätgens, J., Polen, T., 2015. Biosensor-driven adaptive laboratory evolution of L -valine production in Corynebacterium glutamicum. Metab. Eng. 1-11. doi:10.1016/j.ymben.2015.09.017

Michel, A., Koch-Koerfges, A., Krumbach, K., Brocker, M., Bott, M., 2015. Anaerobic growth of Corynebacterium glutamicum via mixed-acid fermentation. Appl. Environ. Microbiol. 81, 7496-7508. doi:10.1128/AEM.02413-15

Moon, M.-W., Park, S.-Y., Choi, S.-K., Lee, J.-K., 2007. The phosphotransferase system of Corynebacterium glutamicum: features of sugar transport and carbon regulation. J. Mol. Microbiol. Biotechnol. 12, 43-50. doi:10.1159/000096458

Ohnishi, J., Mitsuhashi, S., Hayashi, M., Ando, S., Yokoi, H., Ochiai, K., Ikeda, M., 2002. A novel methodology employing Corynebacterium glutamicum genome information to generate a new L-lysine-producing mutant. Appl. Microbiol. Biotechnol. 58, 217-223. doi:10.1007/s00253001-0883-6

Oide, S., Gunji, W., Moteki, Y., Yamamoto, S., Suda, M., Jojima, T., Yukawa, H., Inui, M., 2015. Adaptive laboratory evolution conferred cross-tolerance to thermal and solvent stress to Corynebacterium glutamicum. Appl. Environ. Microbiol. 81, 2284-2298. doi:10.1128/AEM.03973-14

Pauling, J., Röttger, R., Tauch, A., Azevedo, V., Baumbach, J., 2012. CoryneRegNet 6.0--Updated database content, new analysis methods and novel features focusing on community demands. Nucleic Acids Res. 40, D610-4. doi:10.1093/nar/gkr883

Pfeifer, E., Gatgens, C., Polen, T., Frunzke, J., 2017. Adaptive laboratory evolution of Corynebacterium glutamicum towards higher growth rates on glucose minimal medium. Sci. Rep. 7, 16780.

Sambrook J, Fritsch EF, M.T., 1989. Molecular cloning: A laboratory manual. Second edition., Cold Spring Harbor Laboratory Press. Cold Spring Harbor, NY. doi:10.1016/00928674(90)90210-6

Shinfuku, Y., Sorpitiporn, N., Sono, M., Furusawa, C., Hirasawa, T., Shimizu, H., 2009. 
Development and experimental verification of a genome-scale metabolic model for Corynebacterium glutamicum. Microb. Cell Fact. 8, 43. doi:10.1186/1475-2859-8-43

Takeno, S., Takasaki, M., Urabayashi, A., Mimura, A., Muramatsu, T., Mitsuhashi, S., Ikeda, M., 2013. Development of fatty acid-producing Corynebacterium glutamicum strains. Appl. Environ. Microbiol. 79, 6776-83. doi:10.1128/AEM.02003-13

Teramoto, H., Inui, M., Yukawa, H., 2011. Transcriptional regulators of multiple genes involved in carbon metabolism in Corynebacterium glutamicum. J. Biotechnol. doi:10.1016/j.jbiotec.2011.01.016

Toyoda, K., Teramoto, H., Inui, M., Yukawa, H., 2009. Involvement of the LuxR-type transcriptional regulator RamA in regulation of expression of the gapA gene, encoding glyceraldehyde-3-phosphate dehydrogenase of Corynebacterium glutamicum. J. Bacteriol. 191, 968-977. doi:10.1128/JB.01425-08

Unthan, S., Baumgart, M., Radek, A., Herbst, M., Siebert, D., Brühl, N., Bartsch, A., Bott, M., Wiechert, W., Marin, K., Hans, S., Krämer, R., Seibold, G., Frunzke, J., Kalinowski, J., Rückert, C., Wendisch, V.F., Noack, S., 2015. Chassis organism from Corynebacterium glutamicum- a top-down approach to identify and delete irrelevant gene clusters. Biotechnol. J. 10, 290-301. doi:10.1002/biot.201400041

Unthan, S., Grünberger, A., van Ooyen, J., Gätgens, J., Heinrich, J., Paczia, N., Wiechert, W., Kohlheyer, D., Noack, S., 2014. Beyond growth rate 0.6: What drives Corynebacterium glutamicum to higher growth rates in defined medium. Biotechnol. Bioeng. 111, 359-371. doi:10.1002/bit.25103

Wang, Z., Chan, S.H.J., Sudarsan, S., Blank, L.M., Jensen, P.R., Solem, C., 2016. Elucidation of the regulatory role of the fructose operon reveals a novel target for enhancing the NADPH supply in Corynebacterium glutamicum. Metab. Eng. 38, 344-357. doi:10.1016/j.ymben.2016.08.004

Wang, Z., Moslehi-Jenabian, S., Solem, C., Jensen, P.R., 2014. Increased expression of pyruvate carboxylase and biotin protein ligase increases lysine production in a biotin prototrophic Corynebacterium glutamicum strain. Eng. Life Sci. 0, 1-10. doi:10.1002/elsc.201400185

Yamamoto, S., Gunji, W., Suzuki, H., Toda, H., Suda, M., Jojima, T., Inui, M., Yukawa, H., 2012. Overexpression of genes encoding glycolytic enzymes in Corynebacterium glutamicum enhances glucose metabolism and alanine production under oxygen deprivation conditions. Appl. Environ. Microbiol. 78, 4447-57. doi:10.1128/AEM.07998-11

Yu, G., Wang, L.-G., Han, Y., He, Q.-Y., 2012. clusterProfiler: an R package for comparing biological themes among gene clusters. OMICS 16, 284-7. doi:10.1089/omi.2011.0118

Zamboni, N., Fendt, S.-M., Ruhl, M., Sauer, U., 2009. ${ }^{13}$ C-based metabolic flux analysis. Nat. Protoc. 6, 878-892. 